\title{
Essai de reconstitution de l'histoire des armes miniatures de saint Michel depuis leur introduction au Mont jusqu'à leur disparition
}

\section{Armelle Le Huërou}

\section{(2) OpenEdition \\ Journals}

Édition électronique

URL : http://journals.openedition.org/abpo/1424

DOI : 10.4000/abpo.1424

ISBN : 978-2-7535-1490-4

ISSN : 2108-6443

\section{Éditeur}

Presses universitaires de Rennes

\section{Édition imprimée}

Date de publication : 20 juillet 2003

Pagination : 157-187

ISBN : 978-2-86847-874-0

ISSN : 0399-0826

\section{Référence électronique}

Armelle Le Huërou, «Essai de reconstitution de l'histoire des armes miniatures de saint Michel depuis leur introduction au Mont jusqu'à leur disparition », Annales de Bretagne et des Pays de l'Ouest [En ligne], 110-2 | 2003, mis en ligne le 20 juillet 2005, consulté le 20 avril 2019. URL : http://

journals.openedition.org/abpo/1424; DOI : 10.4000/abpo.1424 


\title{
Essai de reconstitution de l'histoire des armes miniatures de saint Michel depuis leur introduction au Mont jusqu'à leur disparition
}

\author{
Armelle LE HuEROU \\ Doctorante, Université de Rennes 2
}

\begin{abstract}
Quoique les reliques dont il est question ici, un glaive et un bouclier miniatures ayant appartenu à l'archange saint Michel, aient fait l'objet de nombreuses descriptions, aussi bien dans la littérature spécifiquement montoise que dans certains ouvrages de portée moins restreinte ${ }^{1}$, leur aspect matériel nous est très mal connu. Conservées au Mont-Saint-Michel jusqu'en 1790, date à laquelle on perd définitivement leur trace physique, ces armes ont toujours été perçues comme une paire indissociable, ainsi que le suggèrent les textes qui les placent systématiquement sur le même plan et les réunissent souvent sous un vocable collectif. Pourtant, elles semblent n'avoir partagé que la modicité de leur taille et leur « matière d'airain ", sans qu'une véritable unité de facture aient par ailleurs justifié leur appariement. Elles sont dépeintes et mentionnées pour la première fois dans le premier quart du XII ${ }^{\mathrm{e}}$ siècle par Baudri de Bourgueil (1145/6-1130), alors archevêque de Dol (1107-1130), dans un texte qui leur est exclusivement consacré, la Relatio de scuto et gladio sancti Michaelis ou Relation sur le glaive et le bouclier de Saint-Michel :
\end{abstract}

"Au Mont-Saint-Michel appelé Tombe, on voit certaines enseignes (insignia) comme guerrières, pour autant moins appropriées au combat qu'à un jeu d'enfant [...]. Ici, on a placé un ancile de très petite envergure, en airain, de forme presque circulaire, léger, peu épais, orné en quatre endroits du signe de la sainte croix ainsi qu'un glaive miniature figuré à la manière d'un vrai : tous deux cependant, à cause de leur petite taille, ne sauraient trouver un emploi à l'usage de la guerre mais sont seulement à l'image d'armes ${ }^{2}$. "

1. Du Monstier, Arthur, Neustria pia, Rouen, 1663, p. 379-381; Boudon, Henry-Marie, La dévotion aux neufs chœurs des saints anges, Paris, 1755 (1 $1^{\text {re }}$ éd. 1680), p. 294-300. Pour les références complètes des ouvrages cités, cf. Annexe, où les témoins écrits sont classés par période.

2. Le texte latin est celui que nous avons établi dans le cadre de notre thèse dont l'objet est d'éditer, traduire et commenter l'œuvre hagiographique de Baudri de Bourgueil 
On le voit, la description qu'il en fait au début de son texte est sommaire. Elle est toutefois partiellement complétée par Guillaume de SaintPair dans son Roman du Mont-Saint-Michel (vers 1155). Sa description du bouclier est la plus achevée qu'on trouve :

"Au milieu il y a une petite boucle [l'umbo, la partie centrale et bombée du bouclier] et elle est rattachée à quatre croix bien niellés sur argent. À leurs bras, il y a bien plus de cent clous à tête d'argent et dont le reste est de cuivre ou de laiton. Entre la croix et la petite boucle, qui est pointue et toute petite, il y a un cercle bien fourni des mêmes clous dont j'ai parlé. Le petit écu a quatre bras qui s'en vont de quatre côtés à partir de ce cercle dont vous venez d'entendre parler : ils furent reliés à la boucle ${ }^{3}$. "

Malheureusement, les vers relatifs au glaive sont perdus. Malheureusement, car, les évocations successives qu'on trouve jusqu'à la date de la disparition des armes miniatures dans la tourmente révolutionnaire n'ajoutent rien aux descriptions de Baudri et de Guillaume de Saint-Pair. Même les témoignages de ceux qui écrivent les avoir vues de leurs yeux, voire manipulées, n'en révèlent pas davantage sur leur apparence. Quant aux dimensions exactes de ces reliques, les témoignages font également défaut pour les préciser. Baudri suggère qu'elles auraient pu convenir à des enfants pour jouer à la guerre et les témoins postérieurs, en recourant à un terme comme pugio (poignard) pour désigner le petit glaive, suggèrent que cette relique en avait la taille.

On n'en sait donc guère davantage, car la réalité matérielle des armes et la dévotion dont elles font l'objet au Mont ont beaucoup moins intéressé les témoins dont nous disposons que leur histoire et leur signification. Le premier d'entre eux, Baudri, a privilégié le récit des circonstances miraculeuses qui conduisirent les armes au Mont-Saint-Michel. Il inaugure, sans le savoir et, sans doute, sans le vouloir, une série de témoignages focalisés sur l'histoire qui précède leur introduction au Mont, événement que presque tous s'accordent à situer à l'époque mythique immédiatement postérieure à la fondation du sanctuaire (708). La plupart de nos témoins signalent bien que les armes archangéliques sont vénérées par les fidèles, mais cette formule n'est jamais ni contextualisée ni développée. Il est pourtant peu probable qu'elles aient connu une égale faveur entre le moment où

(pour le texte et la traduction actuellement disponibles, cf. Annexe) : "In Monte sancti Michaelis qui Tumba dicitur, quedam uidentur insignia quasi bellica, non tamen bello, immo magis ludo infantili [...] accommoda. [...] Ibi siquidem ancile quoddam appositum est corpore paruissimum, materia ereum, forma pene rotundum, tenue non spissum, quatuor in locis signo sancte crucis insignitum, pariterque gladiolus in modum gladii figuratus, que tamen ambo ad nullum pre modicitate sua belli sufficerent usum, sed dumtaxat armoru instar sunt ".

3. Le Roman du Mont Saint-Michel, v. 3516-3531 : " Li escuz est de tel façon/Cum est escu a champiun/Une boclete a el milié/E quatre croiz environ lié/Neielé[e]s bien à argent;/Clous a es braz bien plus de cent,/Qui sunt d'argent el sommeron,/Desoz de coivre ou de laton./Entre la croiz e la boclete,/Qui est aguë e petitete,/A un cerne bien adoubé/D'altretels clous com ai conté./En l'escuet a quatre braz,/Qui s'en iessent par quatre parz/De cel cerne que vos oiez;/En la bocle furent jostez ". 
elles sont arrivées au Mont et, par exemple, le XVII ${ }^{\mathrm{e}}$ siècle, où elles comptent parmi les maîtresses pièces du trésor montois. Après la disparition des armes, les historiens et érudits des $\mathrm{XIX}^{\mathrm{e}}$ et $\mathrm{XX}^{\mathrm{e}}$ siècles ${ }^{4}$ continuent de s'engouffrer dans cette voie et ne s'intéressent donc qu'à ce qui devient la légende du bouclier et de l'épée miniatures. À de rares exceptions près ${ }^{5}$, ils ne remettent pas véritablement en question la date d'introduction proposée par Baudri et fixée par les traditions ultérieures. Il faut attendre Jean Laporte ${ }^{6}$ pour que soit enfin rétablie une partie de la vérité sur l'origine des armes. Si les raisons qui le conduisent à postuler une date plus récente d'introduction de ces reliques sont loin d'être toujours convaincantes, son article très suggestif ouvre des pistes jusqu'alors inexplorées.

L'étude du premier témoignage, la Relatio, permet de saisir les raisons de son succès et le rôle fondateur qu'il joue dans l'élaboration et la transmission de la tradition jusqu'en 1790. Pourtant, la continuité des témoignages n'est qu'apparente et révèle que les armes miniatures n'ont peut-être pas immédiatement connu la faveur attendue pour des reliques archangéliques. S'il n'est pas possible de dater précisément les grandes étapes qui scandent leur présence au Mont, on parvient au moins à en repérer la période d'introduction, les débuts timides qui la suivent et les moments où elles sont en grande faveur, à la fois auprès des religieux montois et du peuple.

\section{Le premier témoignage : la Relatio de scuto et gladio sancti Michaelis de Baudri de Bourgueil ou les raisons d'un succès}

\section{Des circonstances de composition mal connues}

Commençons par le premier témoignage faisant état de l'existence des armes. Sans qu'on sache dater précisément l'époque à laquelle Baudri de Bourgueil a composé cette Relation, on sait de manière sûre qu'elle se situe après l'incendie qui ravagea le Mont le 25 avril 1112 et avant 1130 (date du décès de Baudri). On ne sait pas plus déterminer les circonstances réelles de rédaction, tâche qui s'avère d'autant plus difficile que le texte se présente comme le résultat d'une initiative personnelle. Au moment où il écrit ce texte, Baudri, qui d'ailleurs le rappelle à deux reprises dans le cours de la Relatio, comme si cette indication accréditait la véracité de son propos, est archevêque de Dol (1107-1130). Le siège qu'il occupait le mettait donc nécessairement en rapport avec le Mont-Saint-Michel géographiquement très proche. Par ailleurs, son inclination pour la Normandie, où il finit ses jours et fut enterré à Saint-Samson-sur-Risle, ses relations avec le pouvoir

4. Il suffit de consulter l'ensemble des textes, articles et ouvrages confondus, consacrés à l'histoire de l'abbaye pour en avoir une idée.

5. Musset, Lucien, "Pèlerins et pèlerinages en Normandie ", Annales de Normandie, 12, 1962, p. 130 (il propose de situer leur date d'introduction au Mont au milieu du Ix siècle) et LAPORTE, Jean, "L'épée et le bouclier dits de Saint Michel ", Millénaire Monastique du Mont-Saint-Michel, t. II, Vie montoise et rayonnement intellectuel, Bibliothèque d'histoire et d'archéologie chrétiennes, Paris, 1967, p. 397-410.

6. Op. cit. n. supra. 
laïc normand ${ }^{7}$ dont on sait qu'il s'immisçait alors dans la gestion spirituelle des abbayes et évêchés peuvent avoir favorisé chez Baudri un intérêt particulier pour le sanctuaire normand alors notoirement soumis aux décisions du souverain anglais. Cela dit, aucune mention d'un éventuel commanditaire ou d'un destinataire précis, aucune indication explicite sur les motivations qui animent son auteur ne sont perceptibles dans le texte. Baudri prétend seulement écrire dans le but - méritoire - de préserver des outrages du temps " la noble histoire " des précieuses armes ${ }^{8}$, dont les sources écrites de documentation, les " chartes", viennent d'être " réduites en cendres " dans le récent incendie qui embrasa le Mont ${ }^{9}$. Sans doute cette protestation de bonne foi est-elle partiellement sincère, mais, comme ce topos littéraire - si cher à Baudri qu'on le retrouve dans tous ses textes hagiographiques ${ }^{10}$ - est ici le seul objectif qu'il consente à avouer, il est permis de penser que cette entreprise de conservation de l'histoire des armes, d'authentification et de définition de leur statut - car c'est aussi, voire surtout, de cela qu'il s'agit - répond également à des préoccupations plus prosaïques. Si l'on peut en saisir certaines, beaucoup d'éléments continuent de nous échapper.

La Relatio n'est pas présentée par Baudri comme le premier texte consacré à l'épée et au bouclier, mais comme l'aboutissement et la mise en forme d'une double tradition, écrite et orale, alors en vigueur au Mont - tout au moins jusqu'à l'incendie pour la première. C'est en " auditeur fidèle ", nous dit-il, qu'il consigné le récit qu'a bien voulu lui faire un " conteur fidèle ", le prieur du Mont ${ }^{11}$. Baudri prend soin de préciser que cette source de première main, ce heros comme il l'appelle, est " instruit des arts libéraux et averti des choses du siècle ${ }^{12}$ " et surtout, ce qui est essentiel pour la crédibilité du récit qu'il rapporte, qu'il maîtrise parfaitement les " antiques histoires de l'église " dont il a eu connaissance par les archives du lieu et la tradition orale c'est-à-dire ici les récits dont ses aînés l'ont abreuvé lorsqu'il était enfant ${ }^{13}$. Toutes ces indications ont vocation à légitimer le récit

7. Baudri est en effet l'auteur de plusieurs épîtres poétiques adressées aux filles de Guillaume le Conquérant, dont le poème à la comtesse Adèle de Blois est parmi les plus fameuses. On relève aussi sa présence auprès du duc de Normandie et roi d'Angleterre Henri I ${ }^{e r}$ Beauclerc en qualité de témoin lors de la confirmation de la donation de la forêt de Savigny à l'ermite Vital, le 2 mars 1112 à Avranches (VAN MoolEnBroeK, Jaap, Vital l'Ermite, prédicateur itinérant fondateur de l'abbaye normande de Savigny, Revue de l'Avranchin et du pays de Granville, mars 1991, t. LXVIII, n 346, p. 260-263).

8. Relatio : "nequando nobilis uilescat hystoria, ne forte emula uetustate deleta depereat ".

9. Relatio : «Scripta [...] hec ad nos usque manserunt, sed flammis crepitantibus que nuper in nostra ecclesia furuerunt in fauillam redacta sunt. "

10. Cf. entre autres, Historia Hierosolymitana, PL, t. 166, prol., col. 1064 : " ne invidiae oblivioni cederet historia digna relatu ", "nobilis materies viluerat "; Vita sancti Hugonis, ibid., col. 1163 : " quaelibet nobilis historia, nisi urbane recitetur, vilescit ", etc.

11. Relatio : "Quod uero de his insignibus a fideli relatore fidelis auditor audiui, qualibuscumque litteris inserui $[\ldots] »$.

12. Ibid. : " uirum admodum liberalibus litteris eruditum et de secularibus industrium ».

13. Ibid. : « loci pleniter antiquitates nouerat [...]. Replicabat [...] antiquas ecclesie hystorias, quas uel ab ipsis archiuis cenobii hauserat, seu a prioribus suis olim puer audierat" ". 
du prieur, qui constitue l'essentiel de la Relatio, et, par ricochet, la Relatio elle-même. La fortune que connaîtra le texte de Baudri, considéré par ses successeurs comme la source autorisée de l'histoire des armes de saint Michel, est la meilleure preuve que le dispositif qu'il a adopté a pleinement fonctionné.

\section{Une structure très élaborée}

La structure qui ordonne la Relatio est en effet très élaborée. Le texte original de Baudri se présente comme une première déposition écrite (celle de Baudri) renfermant une seconde déposition (celle du prieur), elle-même construite autour de témoignages antérieurs, écrits et oraux, relatant l'histoire des reliques. Elle est donc organisée autour de la relation du prieur (soit 76 \% de la Relatio), elle-même construite autour de l'histoire du bouclier et de glaive (soit $59 \%$ de la Relatio et $78 \%$ du discours du prieur). On la peut schématiser ainsi :

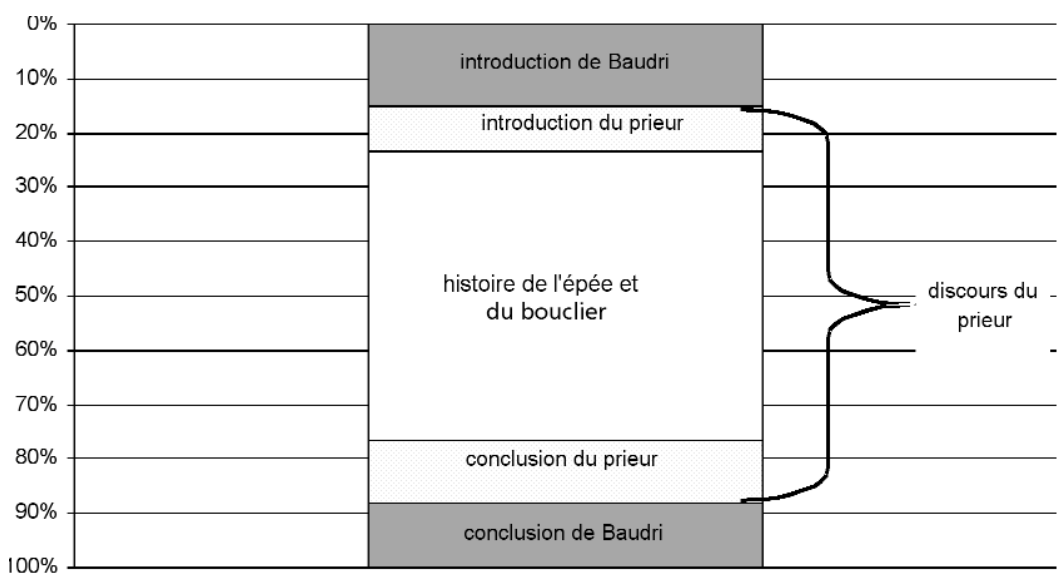

Baudri fait en effet précéder le discours du prieur d'une introduction (10 \% de la Relatio) où, après une brève description des armes miniatures, il se met en scène dans le rôle du visiteur curieux d'en connaître l'histoire. Il introduit ensuite le discours qu'il rapporte en présentant dans les termes flatteurs qu'on a vus son informateur et s'efface pour lui déléguer la parole au style direct. Suit la relation du prieur, nettement découpée, à l'image de la Relatio elle-même, en trois parties. Une introduction décrit brièvement (13\% du discours) le Mont, rappelle les circonstances miraculeuses de sa fondation, évoque son essor immédiat comme centre de pèlerinage et situe - du reste, assez imprécisémment - l'époque et le lieu où se déroulent les événements. Le prieur s'efface à son tour derrière le récit qu'il fait de l'histoire du bouclier et de l'épée miniatures et ne reprend la parole que pour conclure (9\%) en insistant sur la véracité de ses dires - à grand renfort 
d'arguments d'autorité (tradition orale, tradition écrite, foi). Baudri prend alors le relais pour conclure à son tour (13\%) : après avoir répété en les développant et en les illustrant les arguments de son interlocuteur, il expose les motivations qui l'ont poussé à écrire et termine sur un appel à la bienveillance des lecteurs.

\section{Au centre de la Relatio : un récit de translation}

Le récit de l'histoire des armes, doublement encadré par le discours du prieur et celui de Baudri dans lequel il est inséré, acquiert au cœur de cette construction en miroir une certaine autonomie qu'on peut expliquer par sa genèse particulière et le genre littéraire auquel il se rattache. Ainsi que Jean Laporte l'a mis en évidence, pour cette partie de la Relatio, Baudri semble avoir exploité un récit issu d'adaptations successives d'un texte peut-être d'origine égyptienne ${ }^{14}$. En voici l'essentiel : dans une région située par certains manuscrits " à droite " de l'Asie ${ }^{15}$, dans un autre, aux alentours de Siponte ${ }^{16}$, un dragon s'est installé sur une montagne. De là, il empoisonne de son haleine la contrée et contraint les hommes à fuir le pays. Ceux-ci s'adressent alors au Christ et font vœu d'édifier une église s'il les débarrasse du monstre. Le Christ leur délègue Michel qui, sous la forme d'un oiseau armé d'un glaive de feu, tranche la bête en douze morceaux. Le peuple s'acquitte alors de son vœu et élève une église dédiée à l'archange où vivent depuis lors cent cinquante hommes. La comparaison entre le modèle supposé et le récit central de la Relatio établit indéniablement la preuve qu'une parenté thématique et lexicale étroite unit les deux textes (nous y reviendrons), mais elle permet aussi de saisir le travail effectué par Baudri pour conformer son texte aux stéréotypes littéraires en vigueur dans un genre hagiographique particulier, le récit de translation. Le récit central de la Relatio est en effet l'exacte illustration des trois phases identifiées par Martin Heinzelmann comme constitutives de ce genre ${ }^{17}$.

\section{L'invention des armes}

La première partie de ce récit central commence par l'évocation des conditions de la découverte des armes miniatures. À une époque située entre la dédicace de l'église du Mont et la réforme bénédictine (966), dans

14. LAPORTE, Jean "L'épée et le bouclier dits de Saint Michel ", Millénaire Monastique du Mont-Saint-Michel, t. II, Vie montoise et rayonnement intellectuel, Bibliothèque d'histoire et d'archéologie chrétiennes, Paris, 1967, p. 397-410. Nous reviendrons plus loin sur le rôle de Baudri dans l'adaptation d'une des versions de ce texte.

15. Vat. Reg. 703 B, $\mathrm{f}^{\circ} 101$ : "in dexterali parte Asie " (ms reproduit in Orderici Vitalis Historiae Ecclesiasticae libri VII et VIII e codice vaticano reg. 703 A, Paris, 1902).

16. BNF lat. $2873 \mathrm{~A}, \mathrm{f}^{\circ} 109 \mathrm{v}^{\circ}-110$ : Le texte est copié entre l'Apparitio $S$. Michaelis et la Revelatio.

17. Heinzelmann, Martin, Translationsberichte und andere Quellen des Reliquienkultes, Typologie des sources du Moyen Âge occidental, fasc. 33, Brepols, Turnhout, 1979, p. 77-80. 
une région, située vaguement " outre-Angleterre ${ }^{18}$ ", sévit un serpent aux proportions monstrueuses qui menace de famine les contrées voisines. Conscients de leur impuissance, les habitants se tournent vers Dieu et font appel à leur évêque qui leur impose trois jours de jeûne et de prière. " On ${ }^{19}$ " leur enjoint alors de partir tous ensemble le troisième jour combattre la bête. Clercs et laïcs, brandissant reliques et armes, se rendent en procession - et dans l'épouvante - à l'endroit où elle vit. Survient alors un miracle inaugural coïncidant avec la découverte des armes miniatures : alors qu'ils vont assaillir le monstre, ils se rendent compte qu'il est déjà à l'état de cadavre, découpé en tronçons, et découvrent l'épée et le bouclier miniatures. Soupçonnant une intervention divine, ils se tournent à nouveau vers Dieu pour qu'il leur révèle le secret de cet armement manifestement inadapté au combat. Saint Michel apparaît alors à l'évêque pour lui révéler et garantir l'origine, l'authenticité et la destination religieuse et géographique des armes qui acquièrent alors le statut de reliques, sans pour autant que cela soit dit explicitement.

\section{Leur translation depuis le lieu de découverte à l'endroit élu par le saint}

Après une traversée sans encombre, les quatre personnages retenus par l'évêque se mettent en devoir de gagner le Mont Gargan. Au bout de plusieurs jours de marche infructueuse, lassés d'errer, ils se concertent et comprennent que ce n'est pas au mont Gargan que saint Michel les a envoyés, mais plutôt au Mont-Saint-Michel dont ils ont entendu parler et qui se situe tout près d'eux. Avant de se mettre en route, ils préfèrent solliciter l'avis de l'archange. Celui-ci leur apparaît donc et valide leur interprétation, en précisant bien que c'est le Mont-Saint-Michel qu'il a élu comme " résidence secondaire " (sa " résidence principale " étant aux cieux $)^{20}$. L'errance inexplicable de la délégation chargée d'accomplir l'ordre de l'archange, la nouvelle apparition de ce dernier pour confirmer son désir que les armes soient conservées au Mont-Saint-Michel dont il se confirme le patronus sont autant de lieux communs repérables dans l'évocation de nombreuses translations de reliques.

\section{Leur aduentus dans la communauté}

La délégation se rend donc aussitôt au Mont où les reliques sont reçues dans les formes. Ils racontent l'histoire et s'en portent garants (on consigne leurs noms - que le prieur qui les a pourtant " souvent lus ${ }^{21}$ " se garde de donner - dans les chartes du monastère). Les religieux et les " indigènes " se réjouissent et rendent grâce à la légion angélique. Ce dernier épi-

18. Relatio : "ultra Angliam in remotissima quadam regione".

19. Ibid. : "Indictumque est illis ".

20. Ibid. : «[...] locus ille nouiter edificatus ecce diuersorium nostrum est - In celis habemus contubernium, in terris diuersorium ".

21. Ibid. : "cartis $[\ldots]$ que multotiens uidimus et legimus ". 
sode reprend les situations et le lexique de l'adventus des reliques, moment décisif où elles font leur entrée dans la communauté élue par le saint pour y exercer sa protection et son autorité.

\section{Une preuve d'authenticité et d'antiquité plus qu'un simple témoignage}

Comme tout texte de ce genre, ce récit de translation de facture très classique est d'abord ouvertement destiné à promouvoir l'authenticité des armes (reconnues par saint Michel comme siennes et soumises aux étapes qui scandent le destin habituel des reliques), le but avoué étant de convaincre les sceptiques et les incrédules en recourant notamment aux topoi faisant autorité. Les conclusions respectives du prieur et de Baudri qui se réclament de témoignages antérieurs et accumulent les arguments d'autorité viennent alors s'ajouter pour lever définitivement tout soupçon. Ensuite, et c'est là le message principal que délivre la partie centrale de la Relatio, il s'agit d'affirmer et d'officialiser la volonté de l'archange d'être le patron "spé$\mathrm{cial}^{22}$ " du Mont-Saint-Michel au détriment du mont Gargan. Historiquement, le sanctuaire des Pouilles est, comme le rappelle le prieur dans son introduction, l'illustre prédécesseur auquel le mont normand est redevable de ses premières reliques angéliques ${ }^{23}$. Sa légitimité s'est donc acquise dans l'ombre fraternelle du premier grand sanctuaire dédié à l'archange. Le récit même de fondation du Mont, la Revelatio $^{24}$, doit beaucoup à son pendant adriatique, l'Apparitio ${ }^{25}$ auquel il emprunte de nombreux éléments, sans compter que certains choix architecturaux dans l'édification du sanctuaire normand s'inspirent directement de celui du Gargan. Sans remettre ouvertement en cause cette filiation inscrite dans l'histoire et l'architecture du Mont-Saint-Michel, le récit de translation s'emploie à établir la primauté soidisant ancienne du mont normand - puisque datée par le texte de l'époque immédiatement postérieure à sa fondation - sur le mont Gargan. La translation des armes et la péripétie majeure de l'errance des messagers (qui veulent absolument aller à Gargan mais en sont irrésistiblement détournés) matérialise évidemment ce transfert de faveur et de protection michéliennes, ce que corroborent les déclarations tout à fait claires de l'archange. Si l'on se souvient que la Relatio est intégralement construite autour de ce récit de translation, il est évident qu'elle est moins un témoignage anodin qu'une tentative pour imposer efficacement des armes dont on devine qu'elles posaient problème à un certain nombre de personnes.

22. Relatio : "specialius $[\ldots]$ patrocinium ".

23. Ibid. : "Ditata est etiam [ecclesia] pallioli portiuncula quod in Gargano manus angelica preparauerat... ", qui renvoie explicitement à la Revelatio ecclesiae sancti Michaelis dite Revelatio (meilleure édition in Dom T. LE RoY, Curieuses recherches, t. 1, p. 407-419) : "partem [...] rubei pallioli quod ipse memoratus Archanglus in monte Gargano supra altare quod ipse manu construxerat posuit ".

24. Cf. n. supra.

25. Connu également sous le titre Memoriam, son titre complet est Apparitio S. Michaelis in Monte Gargano, (BHL 5948). 
Pour autant, ce n'est pas ce qui a le plus frappé les témoins ultérieurs. Même s'ils se font l'écho des propos de l'archange et des péripéties subies par les messagers chargés de remettre les armes au Mont normand, ils n'y ont pas accordé une importance excessive et ont préféré voir dans le récit rapporté par Baudri un certificat d'authentification des armes et l'explication à la fois matérielle et spirituelle de leur présence au Mont. L'explication matérielle réside bien sûr dans le récit des circonstances de l'introduction des armes au Mont, dont l'essentiel, même déformé, est toujours présent dans les évocations postérieures à Baudri : il est donc toujours question de la grande antiquité des armes et de leur provenance mystérieuse. L'explication religieuse et spirituelle est, quant à elle, tirée des propos mêmes de l'archange qui, lors de sa première apparition, explique que les armes sont une preuve concrète de son intervention contre un ennemi. Il justifie au passage leur taille ridiculement petite : elles n'ont évidemment pas servi à terrasser le serpent mais sont là pour fournir à l'âme humaine un support concret à sa méditation et le sanctuaire bénéficiaire en tirera, en même temps que la preuve de la faveur de l'archange, un moyen de consoler les pèlerins ${ }^{26}$.

Jusqu'alors inexistants, les témoignages sur les armes de saint Michel se multiplient donc après Baudri jusqu'à l'époque qui précède de peu leur disparition matérielle (1790) ${ }^{27}$. Si certains d'entre eux semblent ne présenter avec la Relatio que de vagues similitudes, beaucoup reprennent à leur compte, parfois à leur insu, les données de la source la plus ancienne dont la composition par un archevêque garantit la véracité des données.

\section{L'histoire des armes après la Relatio : une fausse continuité}

\section{Une première reprise de la Relatio sans lendemain}

Dès la fin du XII ${ }^{\mathrm{e}}$ siècle, un texte français en vers, Le roman du Mont-SaintMichel (vers 1155), reprend in extenso la Relatio dont il conserve la structure initiale et les moindres détails. Le seul écart que se permette l'auteur, Guillaume de Saint-Pair (v. 1125-v. 1205), est, on l'a vu, le développement de la description de l'écu et, peut-être, car les vers qui s'y rapportaient sont perdus, celle de l'épée. Il n'est visiblement tributaire que du seul Baudri et quand Baudri est imprécis, il ne cherche pas à proposer une autre interprétation, comme si, au moment où il compose son œuvre, n'existait de l'histoire des armes que la seule version officialisée par le texte de Baudri. Ainsi, dans la Relatio, l'histoire des armes débute dans une "région très reculée outre-Angleterre ". Guillaume de Saint-Pair reprend cette indication, si peu satisfaisante qu'elle soit pour un auditoire curieux, qui devient

26. Saint Michel s'adresse en effet en ces termes à l'évêque : « [...] ista nostra fuit armatura. Non quod armis materialibus uel quibuslibet supplementis indiguerimus, sed ut taliter hebitudini humane satisfecerimus que [...] saltem aliquid uideat per quod fides uestra proficiat et per huiusmodi argumenta pedetentim conualescat. En a laudibus illius nullatenus absistatis qui uos ab inimico uestro uisibili per ministerium nostrum inuisibiliter liberauit ".

27. Cf. Annexe. 
"En un realme outre Engleterre " mais il ajoute " Je ne sei pas nummer la terre $^{28}$ ". Il est tentant de lire dans cet aveu d'ignorance et dans l'absence d'hypothèse pour pallier à l'imprécision de Baudri (ou du prieur, si l'on veut) que les armes ne font l'objet d'aucune rumeur dans l'enceinte du monastère. Il n'existerait pas d'autre tradition que celle fixée par l'archevêque de Dol. Soit que la Relatio ait mis fin à toutes les traditions plus ou moins divergentes qui la précédaient. Soit, plus probable, qu'il n'ait existé aucune tradition relative aux armes de l'archange avant la Relatio, parce qu'elles n'étaient pas encore exposées à la vénération des fidèles. En tout cas, la Relatio est alors la seule source officielle de renseignements.

Il faut ensuite attendre deux siècles au moins avant de trouver à nouveau trace écrite des armes de saint Michel et de leur histoire. Elles n'ont donc pas sombré dans l'oubli, pas plus que leur histoire, dont circulent des versions différentes de celle de la Relatio, peut-être dès le milieu du XIV ${ }^{\mathrm{e}}$ siècle, au moins dès le début du $\mathrm{Xv}^{\mathrm{e}}$ siècle.

\section{Des flottements dans la transmission de la tradition : indices de la vitalité de la Relatio?}

Un Mystère ${ }^{29}$ montois, daté des XIV ${ }^{e}-X V^{e}$ siècles

Un texte copié au milieu du $\mathrm{Xv}^{\mathrm{e}}$ siècle, mais dont la rédaction est située courant XIV ${ }^{\mathrm{e}}$ siècle, au plus tard début XV $\mathrm{V}^{\mathrm{e}}$ siècle, atteste la notoriété de l'épée et du bouclier à cette époque. Ce Mystère composé en français, qui nous est parvenu très mutilé ${ }^{30}$, montre que les armes et leur histoire étaient dignes, dans l'esprit de l'auteur au moins et sans doute dans celui des pèlerins auxquels était probablement destiné ce drame, de figurer à côté des miracles les plus prestigieux du sanctuaire normand ${ }^{31}$. Son caractère fragmentaire ne permet malheureusement pas de mesurer à quel point la tradition rapportée ici s'éloigne ou se confond avec celle de la Relatio, un certain nombre de glissements, voire de divergences sont repérables. Pourtant, la trame du récit est fondamentalement la même : un dragon qui sévit dans une région (ici totalement anonyme) est trouvé mort par le peuple qui s'apprêtait à l'aller assaillir. À ses côtés sont découvertes les " enseignes ", dont personne ne connaît la provenance. L'évêque envoie des messagers les porter au mont Gargan mais, plus ils avancent en direction de leur but, plus ils s'éloignent de cette destination. Saint Michel intervient alors et les dirige

28. Roman, v. 3211-3212, éd. cit. p. 546.

29. Par commodité, nous désignons ainsi le fragment du Mystère découvert en 1861 et édité par Eugène RoBILLARD DE BEAUREPAIRE en 1862 (Cf. annexe) qui évoque les circonstances de sa découverte et la datation du texte (p. 3-4).

30 . Il ne nous en est parvenu que 138 vers qui mettent en scène plusieurs miracles différents, dont aucun n'est complet.

31. Eugène Robillard de Beaurepaire a en effet montré que la scène qui précède celle de l'histoire de l'épée et du glaive a trait au miracle de la femme qui enfante sur la grève à marée montante et qu'une de celles qui suit fait référence à une visite nocturne de l'archange au Mont. 
vers le Mont-Saint-Michel. Certains détails, indiquent même, sinon une réelle connaissance de la version de Baudri, au moins une grande familiarité avec elle. Un des protagonistes du drame, Populus, qui représente les messagers venus apporter les " enseignes ", expose à un de ses interlocuteurs, l'abbé du Mont, les raisons et circonstances de leur venue au Mont. Au moment d'évoquer l'attaque que leur peuple se décide à mener contre le "serpent molt cruel ", il précise que le peuple veut "le tuer ou le chacier ${ }^{32}$ ", alternative identique à celle qui est proposée dans la Relatio où il s'agit d'aller attaquer le serpent pour le " mettre en fuite ou, si quelque stratagème le leur permettait, pour le supprime ${ }^{33}$ ". Le discours de bienvenue de l'abbé à l'adresse de Populus fait également écho à un bref passage de la Relatio. Le prieur du texte de Baudri rapportait que les gens du Mont se rendent tout de suite compte que les messagers sont " des personnes honorables". Pour cette raison, " ils [les gens du Mont] écoutaient attentivement leurs explications [...] : ils ne pouvaient douter d'eux, dont le seul respect qu'ils inspiraient exigeait qu'on les crût ${ }^{34}$ ". L'abbé exprime exactement la même idée : "Volontiers vous escouteron/Quer vous nous semblez gens honnêtes/Par quoi point ne vous doubteron;/Quer gens de bien pert que vous estes. A voir vostre filomie/Ignorer n'en fault nullement ${ }^{35}$." Malgré ces quelques réminiscences et la conservation de la trame essentielle de l'histoire des armes qui renvoient assez précisément à la tradition transmise par la Relatio, le texte du Mystère s'en écarte en de nombreux endroits.

Certaines modifications qu'il enregistre sont d'ailleurs de taille. Dans le désordre et sans prétendre à l'exhaustivité, on peut relever :

- réduction du nombre de messagers chargés d'apporter les enseignes au sanctuaire de l'archange : aux "quatre des notables de ce territoire " délégués par l'évêque chez Baudri sont substitués deux messagers dont Populus ${ }^{36}$;

- provenance géographique des armes inconnue : la région où sévit le dragon ne bénéficie d'aucun éclairage géographique (" au party dont nous sommes ", dit simplement Populus) mais, nouveauté, c'est dans un " maroys " que git le dragon mort;

- " laïcisation " du récit : la figure de l'évêque est reléguée à l'arrièreplan et son rôle se cantonne à envoyer les messagers " au Mont Gargaine de Champaigne ${ }^{37}$ "; le clergé disparaît aussi, tout comme les apparitions de saint Michel, le jeûne et les prières. C'est dans ce sens qu'on peut dire que l'histoire est " laïcisée ", puisque c'est le peuple qui, seul, part à l'as-

32. Ibid., p. 11.

33. Relatio : " ut [...] accederent ad serpentem effugandum, uel si qua possent arte perimendum".

34. Relatio : "Videbant [...] indigene personas honrabiles et attendebant [...] raciocinationes. Non enim eis poterant discredere, quorum dumtaxat credi debebatur reuerentie ".

35. Mystère, p. 11.

36. Relatio : "Electis quatuor de territorii illius primoribus "; Mystère, p. 15.

37. Mystère, p. 15 : «L'eveque n'y sceut qu'aviser/Quant au regart de celle enseigne/Fors porter pour en delivrer/Au Mont de Gargaine en Champaigne ". 
saut du serpent et qui, seul, s'entretient avec un " jouvencel [...]/Disant estre lange Michel $^{38}$ ", le clergé et l'évêque auxquels on fait appel pour élucider le mystère des armes étant incapables de l'élucider ${ }^{39}$;

- datation différente pour l'arrivée des armes au Mont, modification peut-être la plus notable. L'arrivée des armes n'est plus datée de l'époque précédent l'introduction des moines bénédictins (966), mais de la prélature d'un des deux premiers abbés bénédictins. L'interlocuteur de Populus se nomme "Mainart Abbas Montis ", sans qu'on puisse décider s'il s'agit de l'oncle Mainart I (966-991) ou du neveu Mainard II (991-1009).

Qu'on ait restreint le nombre des messagers peut s'expliquer par des contraintes liées à la mise en scène du récit; la " laïcisation " de l'histoire aussi : populariser des miracles dont le Mont avait été le théâtre et le bénéficiaire impliquait qu'on prît en compte les attentes du public de pèlerins. La localisation de l'invention des armes dans un marais et la datation plus récente de leur arrivée au Mont ne sauraient être le fruit de telles préoccupations. En revanche, il y a pu avoir contamination, volontaire ou non, entre les données de la Relatio et celles d'un miracle qui présente avec elle certaines similitudes thématiques et structurelles frappantes. Intitulé $D e$ repertione sanctarum reliquiarum ${ }^{40}$, il raconte comment, après le premier incendie qui ravagea le Mont (992) sous la prélature de Mainard II, la communauté rentra en possession de la boîte contenant les reliques en provenance du mont Gargan. Quatre éléments au moins peuvent avoir prêté à confusion : a) l'incendie, parce qu'il est l'occasion de la disparition des reliques qui présidèrent à la fondation du Mont (dans la Relatio, l'incendie détruisait les documents originaux qui témoignent de l'authenticité des armes); b) la décision de l'abbé et des moines de " de notifier trois jours de jeûne accompagné d'une très grande contrition du cœur et d'aumônes ${ }^{41}$ " pour que " tout le peuple de cet endroit prie " pour que lui soient rendues les reliques; c) l'issue de ce jeûne de trois jours : leur prière est exaucée et, le troisième jour, un pêcheur découvre la boîte au pied du Mont; d) leur commune origine archangélique.

Il n'est pas inconcevable que les armes de saint Michel et les reliques du mont Gargan, toutes deux victimes d'un incendie - quoiqu'à des degrés différents -, toutes deux découvertes à l'issue d'un jeûne de trois jours imposé à la population locale se soient trouvées réunies dans une même

38. Mystère, p. 15.

39. Ibid., p. 12 : «Y donc si sage n'y aveit/De nostre eveque ou gens d'eglise/Qui sachent qui avoit frapée/Ceste beste [...]."

40. Miracle tiré de l'Introductio monachorum (BHL 5952), récit de l'histoire de l'abbaye à partir de l'arrivée des Bénédictins, conçu comme la suite de la Revelatio. Édition dans Thomas LE RoY, Curieuses recherches, t. 1, p. 419-465. Le De Repertione sanctarum reliquiarum est aux p. 439-442. Les BM Avranches ms 211, 212, en conservent le texte latin ainsi que le ms 213 où ce miracle est copié immédiatement à la suite de la Relatio (f $\mathrm{f}^{\circ} 31 \mathrm{v}^{\circ}-33$ ).

41. BM Avranches, ms 213, $\mathrm{f}^{\circ} 32$ : « decreuerunt cum summa cordis contritione elemosinarumque largitione triduanum indicere ieiunium, quo spacio cuncta ipsius loci plebs [...] exoraret $[\ldots]$ sacrum sibi reddi patrimonium $[\ldots] "$. 
histoire. Les écarts entre la version transmise par Baudri et celle que propose le mystère apparaissent donc surtout comme les altérations normalement subies par un texte qui ne circule plus seulement de manuscrit à manuscrit (Guillaume de Saint-Pair avait, lui, la Relatio sous les yeux) mais est transmis par des récits oraux. Ils sont même les signes d'une certaine vitalité dans la transmission de la Relatio : si la tradition évolue, innove, c'est parce qu'on se l'est appropriée, qu'on se la raconte et qu'on la représente. Elle n'est plus fossilisée dans un manuscrit réservé à quelques rares pèlerins et moines capables de lire.

\section{Un cantique allemand (vers 1457)}

Quoi qu'il en soit, cette version reste tout à fait isolée dans le corpus de textes faisant état de l'histoire des armes. Nous ignorons donc tout de son écho auprès des pèlerins ou à l'intérieur même du monastère, comme nous ne savons rien des circonstances de représentation du mystère (à quand remonte la première représentation? Combien de temps a-t-on joué ce drame? À quelle occasion? etc.). S'il était encore représenté au milieu du Xve siècle, époque à laquelle il a été copié, il n'a en tout cas pas influencé un curieux texte composé sans doute au cours de l'année $1457^{42}$ qui, sans dire un mot des armes de saint Michel, a recours à leur histoire telle que la raconte Baudri pour expliquer la fondation de l'église du Mont. Intitulé De peregrinacione puerorum, ce cantique de 22 strophes conserve la mémoire du pèlerinage qu'effectuèrent de jeunes Allemands cette annéelà ${ }^{43}$. Les huit premières strophes, sur lesquelles nous ne reviendrons pas, retracent les circonstances de leur départ et de leur voyage. Elles sont suivies de trois autres évoquant le Mont et son " temple tout à fait magnifique " où « le prince de la multitude angélique est vénéré comme patron ${ }^{44}$ ". Puis le cantique déroule sur les dix strophes restantes le récit de la fondation de ce " temple", dont on appréciera les similitudes avec le récit central de la Relatio. "Dans les marécages de cet endroit [le Mont], avant les années neuf cents, se terrait un dragon enragé et dont le corps était immense ${ }^{45}$ "; " son venin empoisonnait l'air et précipitait dans la male mort un nombre incalculable de gens ${ }^{46}$ ". Mais, " un jeûne est imposé ${ }^{47}$ ", on implore le

42. Une vague de pèlerinages allemands eut lieu au Mont entre 1450 et 1460, ce dont les annales du Mont (pour l'année 1457, cf. HuYNEs, Histoire générale, t. 1, p. 123-124) mais aussi des chroniques allemandes ont conservé le souvenir (Cf. DuPONT, Étienne, "Les pèlerinages d'enfants allemands au Mont Saint-Michel ", Annales de la société historique et archéologique de l'arrondissement de Saint-Malo, 1906, p. 19-60.).

43. Ibid., p. 34-36.

44. Ibid., p. 35 : «In templo premagnifice/quod tibi decoratur/turbe princeps angelice/patronus veneratur".

45. Ibid. : "In hujus loci palustribus/pre annis nuningentis/Draco latebat turbidus/Et corporis ingentis ".

46. Ibid. : " Ejusdem veneficium/auram intoxicaret/Innumeros que plebium/mala morte prostraret".

47. Ibid. : "Indicitur jejunium ". 
secours de la divinité, initiatives couronnées par l'apparition presque immédiate du " porte-enseigne de la milice céleste ${ }^{48}$ " qui promet l'expulsion du monstre. Toute la communauté sort donc, "l'évêque avec tout le clergé ${ }^{49}$ ", " les petits enfants, le groupe des veuves et le peuple sans armes " qui, en tête, chantent ${ }^{50}$, tandis que "les hommes forts et courageux ${ }^{51}$ " ont revêtu leurs armes. Tous ne désirent qu'une chose : la mort du dragon. Cependant, plus on s'approche, moins on avance et "leurs forces abandonnent les hommes ${ }^{52}$ ". Pourtant, l'un d'eux finit par s'approcher, " mais la bête avide de sang avait déjà quitté la vie ${ }^{53}$ ". Jusque-là, le chant de pèlerinage semble puiser dans la tradition véhiculée par la Relatio. Ensuite, il prend une nouvelle orientation et sa chute n'a plus rien à voir avec la Relatio : tout le monde s'en retourne dans la joie, on loue la Vierge Marie et, le cantique s'achève sur cette strophe : "On fait l'ascension du Mont et, sur l'avis des cieux, on construit la basilique au chef des archanges ${ }^{54}$."

Dans les deux cas, la mort du dragon est l'occasion d'un signe concret de la présence de l'archange au Mont et de la protection qu'il lui accorde. $\mathrm{Au}$ fond, seul change véritablement l'épilogue qui modifie la nature de la trace matérielle commémorant l'intervention de saint Michel en substituant aux armes la "basilique ". Cette dissociation entre l'histoire du dragon et celle des armes et la confusion entre la fondation de l'église du Mont et l'histoire d'un dragon restent un phénomène isolé dans l'histoire du Mont. En revanche, le texte identifié comme une des sources du récit de translation dans la Relatio associe bien la mort du dragon à la fondation d'une église : les habitants de la région annexée par le dragon promettent en effet à Dieu " d'édifier un temple en son nom [...] s'il chasse loin d'eux ce dragon ${ }^{55}$ ". Après l'intervention de l'archange qui élimine la bête et la coupe en douze morceaux, " ce peuple, conformément à sa promesse, construisit une église de grande envergure au nom de Dieu et en l'honneur de saint Michel ${ }^{56}$ ". Qu'ont entendu les jeunes pèlerins allemands? Ont-ils seulement mal compris les récits qu'on leur a fait de l'histoire du Mont? Ontils mêlé deux histoires différentes mais présentant des analogies? la tradition relative aux armes s'est-elle complètement corrompue?

48. Ibid. : "Comparuit militie/mox signifer celestis ".

49. Ibid. : "Episcopus [...] cum toto clerico ".

50. Ibid., p. 36 : "Precinebant pueruli/cetusque viduarum/inermes quoque populi/carmen deo preclarum".

51. Ibid. : "Viri fortes et strenui/armis se decorabant".

52. Ibid. : "Nec vires erant viris".

53. Ibid. : "Sed percruenta bestia/dudum vita recessit".

54. Ibid. : "Prefatus mons ascenditur/et nutu supernorum/Basilice construitur/Duci archangelorum".

55. LAPORTE, Jean, op. cit., p. 409 : «Incolae [...] multa Deo promittebant vota, dicentes quod in nomine eius templum aedificarent cum magna honorificentia, si hunc draconem [...] ab eis expelleret."

56. Ibid., p. 410 : " ecclesiam magne structure in Dei nomine sub honore sancti Michaëlis gens illa juxta promissionem construxit". 


\section{Le ms 212 : une entreprise de vulgarisation réussie}

Nous n'en savons rien. Ce qui est sûr, c'est qu'après 1457, les religieux se préoccupent de vulgariser les circonstances de l'invention des armes et remettent à l'honneur la tradition transmise par la Relatio. En témoigne la rédaction du ms 212, qui fixe, dans la seconde partie du Xve siècle, en français et en latin, c'est-à-dire pour toucher un plus grand public, les traditions en vigueur au Mont. Il semble que certaines vérités autorisées avaient besoin d'être rétablies auprès des moines et du public, en particulier celles qui concernaient les armes. Certes, deux manuscrits, habituellement datés de l'abbatiat de Pierre Le Roy (1386-1411), c'est-à-dire peu antérieurs au Cantique et au ms 212, proposaient in extenso la Relatio de Baudri ${ }^{57}$, mais sa trop grande complexité et, peut-être, surtout, les discours de Baudri et du prieur, s'opposaient à sa diffusion auprès d'un public non averti et peut-être pas forcément acquis. En tout cas, alors qu'ont été mises au net quelques années auparavant les principales traditions du Mont, le ms 212 est entrepris pour rendre les sources historiques et les garanties de l'élection du sanctuaire plus accessibles.

\section{Le De scuto et ense : une recension brève de la Relatio de Baudri}

Connu également sous le titre Varia ad historiam Montis Sancti Michaelis spectantia ${ }^{58}$, copié sous la prélature de Guillaume d'Estouteville (1444-1486), probablement peu après 1457, au moins pour la partie qui nous concerne, ce manuscrit bilingue contient notamment, dans une première partie en latin, les récits abrégés de la Revelatio (f $\left.{ }^{\circ} 1-6\right)$ et de la Relatio $\left(\mathrm{f}^{\circ} 6 \mathrm{v}^{\circ}-10\right)$, suivis de la liste des principaux miracles qui illustrèrent le Mont. La seconde partie débute sur la reprise en français des deux récits abrégés, suivis cette fois de la liste des indulgences octroyées au Mont. Intitulé ici De scuto et ense $S$. Michaelis, le texte latin relatif aux armes de saint Michel s'avère être une recension brève du texte original de Baudri. Son statut ambigu est perceptible dès le titre qui enregistre l'intervention du scribe anonyme du $\mathrm{XV}^{\mathrm{e}}$ siècle et reflète parfaitement les inflexions qu'il a données à la Relatio. Ainsi, la suppression du terme Relatio n'est pas innocente, loin s'en faut : n'ont été retenus de la Relatio que le récit central (le récit de translation) et les premières lignes par lesquelles Baudri débutait sa Relation c'est-à-dire la description des armes. Par ailleurs, le choix de substituer ensis à gladium semble indiquer une volonté délibérée de se démarquer du modèle, dont rien, ni le titre, ni le contenu, ne permet de deviner l'auteur ou la période de rédaction. Toute référence à Baudri, qui, on s'en souvient, dans son texte, parlait à la première personne et faisait mention de sa qualité d'archevêque, a disparu; de même, il ne subsiste aucune allusion à son informateur, le

57. BM Avranches, $\mathrm{ms} 211, \mathrm{f}^{\circ} 26-31 \mathrm{v}^{\circ}$ et $\mathrm{ms} 213, \mathrm{f}^{\circ} 150-153$.

58. Pour une description du ms, voir OMont, Henri, Catalogue général des manuscrits des bibliothèques de France, Départements, t. X, Paris, 1889, p. 95-97; VAn DER Straeten, Joseph, " Manuscrits hagiographiques du Mont-Saint-Michel conservés à Avranches ", Analecta Bollandiana, t. 86, 1968, p. 133. 
prieur. Le texte est présenté comme établi à partir de chartes signées par les messagers porteurs des armes. En ôtant au texte son ancrage dans le $\mathrm{XII}^{\mathrm{e}}$ siècle et en y effaçant les marques du discours, le De scuto et ense est donc donné comme le compte-rendu composé immédiatement après les événements. Pour le reste, le texte retenu par le scribe anonyme est assez conforme à celui que proposent les autres manuscrits de la Relatio, à quelques transitions près : il fallait bien que le scribe anonyme du $\mathrm{Xv}^{\mathrm{e}}$ siècle assurât à son remaniement cohérence et cohésion.

De l'escu et de l'espée S. Michiel :

adapatation française du De scuto et ense

C'est ce texte qu'on retrouve traduit en français aux $\mathrm{f}^{\circ}$ 49-54 du même manuscrit 212 sous le titre De l'escu et de l'espée saint Michiel. On sait que cette version française connut un grand succès et assura la diffusion de l'histoire des armes. Deux siècles après sa rédaction, c'est elle qu'exploitent encore, à leur insu parfois, certains auteurs, quand ils doivent traiter des armes miniatures de l'archange. Même lorsqu'ils écrivent en latin, leur source est aisément identifiable, car ils reproduisent sans broncher le contresens commis dans l'adaptation française du Xve siècle. La Relatio de Baudri précisait qu'au moment d'attaquer le serpent, ses assaillants « se précipitent sur cette charogne puante et impie voient que celui qu'ils craignaient en vie et dont ils craignaient qu'il ne survive est mort et découpé en morceaux ${ }^{59}$ ". L'abrégé latin à l'origine de cette version française offre ici une variante ${ }^{60}$ dont le traducteur anonyme du $\mathrm{Xv}^{\mathrm{e}}$ siècle a rendu compte ainsi : ils « s'assemblèrent sur celle morte chaire venimeuse et horrible, craignans et doubtans qu'il fust encore vif, et le détrenchèrent pièce à pièce ${ }^{61}$ ".

\section{Banalisation d'une histoire des armes informée par de nouveaux éléments (XVII siècle)}

\section{Le succès d'un contresens}

On retrouve en 1604 cette interprétation dans un livret à l'usage des pèlerins montois, l'Histoire de la Fondation de l'Église et Abbaie du Mont S. Michel pres celui de tombe : \& des miracles, reliques, \& indulgences donnez en icelle. Tout recueilli des Archives du dit lieu ${ }^{62}$. Composé par le cordelier F. Feuardent à des fins d'édification et de promotion du sanctuaire normand, cet opuscule de 55 pages consiste essentiellement en une réécriture de la partie française du ms 212, auquel le cordelier a ajouté quelques miracles récents ${ }^{63}$.

59. Relatio : "Irruunt super illud uirosum nefandumque morticinum, et quem metuebant superstitem et uiuum uident exanimem et frustatim detruncatum ».

60. "quem intuebant" au lieu de "quem metuebant".

61. $\mathrm{ms} 212, \mathrm{f}^{\circ} 51$.

62. Cf. Annexe.

63. Le livret est divisé en trois parties : fondation du Mont, indulgences, miracles. Les deux premières parties correspondent exactement aux $\mathrm{f}^{\circ} 45-59$ du ms 212. 
L'histoire des armes y est en bonne place, puisque le livret de pèlerinage lui réserve les pages 26 à 41, soit un peu plus d'un quart de l'ouvrage et l'on y retrouve le fameux contresens : les victimes du serpent "s'en approchent, $\&$ le trouvent tout froid \& roide mort, le tranchent en mille pièces ${ }^{64}$ ". Plus tard dans le siècle, deux éminents savants, Michel Naveau ${ }^{65}$ et Artus du Monstier ${ }^{66}$, se rapportent à l'ouvrage de Feuardent, quand ils relatent l'histoire des armes de saint Michel et reproduisent, en latin cette fois, la même erreur. Le premier, qui se réfère explicitement à Feuardent et semble ignorer l'existence de Baudri ${ }^{67}$, traduit donc littéralement le propos du cordelier, soit : " ad quem [serpentem] accedunt, frigidumque ac mortuum in mille frusta dissecant ${ }^{68}$ " (les assaillants " s'approchent de lui [le serpent] et, comme il est froid et mort, le tranchent en mille morceaux "). Le second cite en marge "Naveau, lib. III, cap. XXI, Feuardent et Baudri" : il faut comprendre qu'à quelques ajouts personnels près (il a quand même vu et manipulé les armes, ce qu'il signale, sans en dire davantage ${ }^{69}$ ), son texte est la copie du passage que Naveau consacre aux armes de saint Michel.

La présence du contresens permet donc de comprendre pourquoi et comment un groupe de textes offre une particularité commune. Il est aussi l'occasion de mesurer l'impact de la version française. Devenue la version officielle et immédiatement accessible de l'histoire des armes de l'archange, elle dispense de recourir au texte latin qu'elle adapte et, à plus forte raison, à la version originale de Baudri. Le seul à le citer, Du Monstier, n'en fait pas usage; Naveau ne cite que Feuardent comme source et ce dernier, qui ne fait aucune allusion à Baudri, semble ne pas avoir su qu'il était à l'origine du texte qu'il reprenait. En revanche, il a ajouté une nouvelle modification à l'histoire des armes et propose de localiser leur invention en Irlande : pour lui, les messagers qui apportèrent les enseignes au Mont sont des " insulaires ${ }^{70}$ ", " homes honorables venus d'un païs outre Angleterre (on pense d'Irlande, dite, Hibernie) ${ }^{71}$ ". Cette assertion, évidemment reprise par Naveau et Du Monstier, n'est bien sûr pas de son cru.

\section{L'origine irlandaise des armes}

Il semble qu'elle se soit imposée comme allant de soi grâce à des lamelles de cuivre attachées à l'écu dont le ms 213 (f¹74) a relevé l'inscription qui y était gravée au début du $\mathrm{XV}^{\mathrm{e}}$ siècle ${ }^{72}$. On les supposait d'une antiquité

64. FEUARDENT, p. 32.

65. Cf. Annexe. NAveau, p. 213.

66. Cf. Annexe.

67. Il a rédigé les chapitres relatifs au Mont, écrit-il, « iuxta relationem F. Feuardentis $S$. Th. Doctoris ex archiuis eiusdem loci " (p. 204).

68. NAVEAU, p. 215.

69. Neustria Pia, p. 379 : « [arma] quae \& ego illic uidi, manibusque meis pertractaui ».

70. FEUARDENT, p. 32.

71. FEUARDENT, p. 28.

72. Le premier écho de cette tradition se retrouve au XVI ${ }^{\mathrm{e}}$ siècle dans l'œuvre - inédite de Robert Cénalis, p. 25 (cf. Annexe). 
antérieure au témoignage de Baudri ou " pour le moins vers ce temps-là ". C'est ce qu'assure Dom Huynes (1640) qui a recopié le texte latin de l'inscription et la traduit ainsi : " Regardez icy (le serpent estant tué) l'escusson de S. Michel, lequel par un miracle qui a esté veu monstre les miracles et de quelle masse l'Irlande a esté jusques à present vilainement affligée par un serpent qui troubloit par les maux qu'il faisoit une populace toute baignée en larmes ${ }^{73}$. " C'est aussi ce dont devait être suffisamment persuadé F. Feuardent (1604) pour ajouter dans son remaniement du ms 212 la précision, entre parenthèses, que « certains pensent [que les armes viennent] d'Irlande, dite, Hibernie ${ }^{74}$ ". Le problème est qu'on voit mal pourquoi, dans cette hypothèse, Baudri et Guillaume de Saint-Pair se sont contentés de situer vaguement quelque part " outre-Angleterre " l'origine des armes miniatures, si les lamelles existaient à leur époque ${ }^{75}$. En revanche, il est aisé de comprendre pourquoi l'on a pu, sans doute fin XIV siècle-début $\mathrm{XV}^{\mathrm{e}}$ siècle, matérialiser par un écrit associé aux armes (un authentique, en quelque sorte) une interprétation, somme toute banale et identifier la région " outre-Angleterre ", gouvernée par le roi Elga (dont il est inutile de préciser qu'on ne trouve aucune trace) à l'Irlande.

\section{L'ultime version : la contribution des Mauristes}

$\mathrm{Au} \mathrm{XVII}^{\mathrm{e}}$ siècle, l'histoire des armes de saint Michel tombe donc en quelque sorte dans le domaine public, puisqu'elle n'est plus l'apanage exclusif d'une littérature spécifiquement montoise (entendre par-là écrite pour le Mont et/ou par des gens qui en sont proches), ainsi qu'en témoignent les ouvrages de Naveau et Du Monstier ou encore celui d'un dénommé Boudon, archidiacre d'Évreux. Venu au Mont en 1667, il y a vu les armes de saint Michel et évoque leur histoire dans son ouvrage de piété consacré à La dévotion aux neufs chours des saints anges, et en particulier aux saints anges gardiens ${ }^{76}$. Il s'attarde alors à faire le récit de l'histoire des armes pour fournir une raison supplémentaire de se recommander à l'archange et d'aller en pèlerinage au Mont. En même temps qu'on assiste à cette banalisation de l'histoire des armes, au Mont même, rattaché depuis 1622 à la Congrégation de Saint-Maur, les religieux entreprennent d'écrire l'histoire de leur abbaye.

73. Histoire générale, t. 1, p. 146. Voici le texte latin qu'il a relevé : « Hic involutum Michaelis cernite scutum/Quod per serpentem turbantem per mala flentem/Plebem, qua massa fuit hûc Irlandia passa/(ce vers ne paroit plus)/ Turpiter occiso monstrat miracula viso ".

74. FEUARDENT, p. 28.

75. Guillaume de Saint-Pair précise même "je ne sei pas nummer la terre " (3212). La datation haute (1130) proposée par Jean LAPORTE (op. cit., p. 398) ne semble donc pas recevable.

76. Cf. Annexe. Il ne cite aucune référence et aucune allusion ne permet de savoir d'où il tire ses informations. On est porté à croire qu'il les obtint de vive voix lors de sa visite au Mont, puisqu'il attribue aux armes une provenance inédite : la Grande-Bretagne. 
Les travaux les plus connus qui nous sont parvenus sont ceux de Dom Huynes et Dom Le Roy, auteurs respectivement de l'Histoire générale de l'abbaye du Mont-Saint-Michel au péril de la mer (1638) ${ }^{77}$ et des Curieuses recherches du Mont Saint-Michel (1648) ${ }^{78}$, deux ouvrages publiés tardivement (fin $\mathrm{XIX}^{\mathrm{e}}$ siècle) par les soins de E. Robillard de Beaurepaire. La qualité même de leurs travaux (documentés aux archives de la communauté, reproduisant des extraits des manuscrits consultés, mais aussi s'appuyant sur des ouvrages disponibles sur les questions qu'ils abordent, etc.), malgré une absence de critique des sources, garantissent aux informations qu'ils délivrent une incontestable autorité. Ainsi, comme le fait remarquer F. Vandenbroucke dans un article consacré aux deux historiens mauristes, " après eux, les sources étant explorées, vont se multiplier les notices et les "histoires" du Mont Saint-Michel ${ }^{79}$ ". C'est particulièrement vrai en ce qui concerne l'histoire de l'écu et de l'épée telle qu'en rend compte Dom Huynes. À partir de tous les manuscrits à sa disposition, y compris la version française du ms 212, dont il donne les références précises ${ }^{80}$, il propose presque intégralement le « tesmoignage de ce très-docte personnage Baldric ${ }^{81}$ ». D'après lui, la seule autorité du prélat " qui ne se contenta point seulement d'adjouster foy à ce qu'il entendoit, mais aussy confirma le tout et en insera son sentiment dans ses escripts $^{82}$ " suffit à garantir l'authenticité des faits consignés. La seule addition qu'il s'autorise est la localisation irlandaise de l'histoire, attestée par " les lames de cuivre attachées à l'escusson [...] posées auparavant le susdit tesmoignage [celui de Baudri] ou pour le moins vers ce temps-là, selon qu'il appert assez par l'antiquité des caractères ${ }^{83}$ " qu'il cite et traduit.

Comme on le voit, Dom Huynes accorde une confiance absolue aux sources conservées à l'abbaye : dès l'instant où elles revêtent une certaine antiquité et offrent des gages d'authenticité, le contenu ne doit pas être remis en question. En accordant une place de choix à l'histoire des armes et en produisant le texte latin dans son intégralité (ou presque) - ce qu'il ne fait pas de manière systématique -, il entend apparemment rétablir la véritable histoire des armes et rendre à son premier témoin la paternité du texte qui la renferme, corrigeant ainsi le contresens véhiculé entre autres par le livret de pèlerinage de Feuardent (qu'il connaît). Sans doute aussi veut-il couper court aux élucubrations de ceux qui " se forgent des chimères en leur esprit " ou sont incrédules ${ }^{84}$ et leur fournir des arguments d'au-

77. Cf. Annexe.

78. Ibid.

79. VANDENBROUCKE, François, « Dom Jean Huynes et Dom Thomas Le Roy, historiens mauristes du Mont Saint-Michel ", Millénaire Monastique du Mont Saint-Michel, Paris, Lethielleux, 1967, t. 2, p. 155-167.

80. Les manuscrits en question sont les mêmes que ceux dont nous disposons actuellement pour établir le texte de Baudri (Avranches BM 211, $\mathrm{f}^{\circ} 26-31 \mathrm{v}^{\circ} ; 212, \mathrm{f}^{\circ} 6 \mathrm{v}^{\circ}-10$ et $\left.\mathrm{f}^{\circ} 49-54 ; 213, \mathrm{f}^{\circ} 150-153\right)$.

81. Histoire générale, t. 1, p. 146.

82. Ibid., p. 137.

83. Ibid., p. 146.

84. Ibid., p. 147. 
torité à ses yeux imparables. Enfin, c'est également un moyen de donner la preuve de ce qu'il avance ailleurs dans son Histoire car il y utilise les données de la Relatio. Le titre du chapitre consacré l'essor rapide du Mont comme lieu de pèlerinage, "La renommée de ce Mont volle de tous costez : le Pape, le Roy de France et les Hybernois y envoient des sainctes reliques, et ce Mont change de nom ${ }^{85}$ ", est explicite, mais moins que son contenu. Après avoir recensé les envois de reliques du pape et de Childebert II, Dom Huynes signale que "St Michel, qui avoit promis à St Aubert d'habiter en ce lieu et d'en avoir soin, excita les Hybernois d'y venir en pèlerinage et d'en apporter aussi [des reliques] " et de raconter brièvement l'histoire des armes en précisant " ainsy que nous dirons plus amplement à la fin du traicté second ${ }^{86} "$.

C'est sous son impulsion que l'histoire des armes acquiert alors sa forme ultime. Ses continuateurs et ses émules ${ }^{87}$ s'en tiendront à ses conclusions, si dénuées de critique fussent-elles, et considèreront toujours Baudri comme la source la plus ancienne et la plus autorisée. Dom Le Roy, le premier, s'en sert pour élaborer la chronologie qu'il propose de l'histoire de l'abbaye et, sur la foi du travail de dom Huynes, situe l'arrivée des reliques au Mont juste après le pèlerinage de Childebert ${ }^{88}$. Toujours d'après Dom Huynes - qui déduisait cette date des propos mêmes de Baudri -, il signale le pèlerinage de l'archevêque de Dol en 1112 au Mont ${ }^{89}$.

Même l'auteur de l'anonyme Histoire Du Mont S.-Michel Depuis sa fondation Par S. Aubert en 708 Jusqu'à L'année $1744^{90}$ qui n'accorde qu'une mention très brève aux armes et donne une autre interprétation des faits s'appuie sur Dom Huynes pour écrire : "Quelques temps après [la venue de Childebert], les Irlandais, sous le règne d'Elga leur Prince, ayant été délivrés d'un épouvantable Dragon par l'intercession de S. Michel auquel ils s'estoient recommandés, y envoyèrent en reconnaissance un écusson et un poignard qui furent trouvés auprès du cadavre de ce monstrueux animal $^{91}$. "Pour ce religieux du XvIII ${ }^{e}$ siècle, les armes de saint Michel ne semblent plus signifier grand-chose : il n'y voit qu'un banal ex-voto d'origine irlandaise et une preuve de la renommée immédiate du Mont après sa fondation. Il ne paraît pas en savoir davantage, ainsi que l'emploi de l'article indéfini (" un poignard ", " un écusson ") peut le suggérer. Certes, son laconisme sur l'histoire des armes, dont ne parvient plus qu'une bribe faussée, est symptomatique de la période où il compose son essai historique (1744), mais cela nous intéresse moins que ce qu'il permet de saisir du statut des armes à cette époque. Il ne reste plus rien des reliques guerrières de l'ar-

85. Ibid., p. 42.

86. Ibid., p. 43.

87. Au nombre desquels, on peut compter la plupart des érudits et historiens du XIX ${ }^{\mathrm{e}}$ siècle.

88. Curieuses recherches..., t. 1, p. 87.

89. Ibid., p. 141.

90. Cf. Annexe.

91. Op. cit., p. 25. (Nous avons normalisé l'orthographe et la ponctuation.) 
change qui méritaient les honneurs d'un livret de pèlerinage au début du siècle précédent. Sans doute encore conservées dans une des " trois grandes armoires " pleines de reliques dont le bénédictin dédaigne d'ouvrir les portes pour en livrer le contenu ${ }^{92}$, il ne leur reste plus qu'à disparaître pour de bon en 1790 en qualité de « bouclier de S. Michel avec son sabre provenant du Mont-Gargan ${ }^{93}$ ". Un siècle après Dom Huynes, il ne reste donc qu'une vague idée de ce que furent les armes de l'archange pour le Mont.

Si l'on revient maintenant sur les aléas que connut la transmission de cette histoire, on est forcé d'admettre que le rôle de Baudri est fondateur. Elle a beau enregistrer à certains moments de sa " carrière " des écarts notables par rapport au texte de la Relatio, ceux-ci demeurent isolés et sans véritable suite. Entre Guillaume de Saint-Pair, qui écrit peu après Baudri, et Dom Huynes qui en fixe durablement les données, la tradition s'est seulement enrichie d'une précision : la provenance irlandaise des armes d'ailleurs probablement directement tirée de la Relatio au XIV ${ }^{\mathrm{e}}$ siècle. Grâce, entre autres, à la mécanique d'un texte conçu comme un témoignage à trois niveaux (celui de l'auteur incluant celui du prieur qui reprend les témoignages écrits et oraux qu'il connaît), l'archevêque de Dol détient le monopole des informations disponibles sur les armes. À l'évidence pourtant, si la Relatio reste le document indépassable sur les armes miniatures, c'est parce qu'il n'y a rien avant. Pour en savoir davantage, il faut donc réexaminer sur nouveaux frais la Relatio, à laquelle tous les témoignages ultérieurs se rattachent de près ou de loin.

\section{La véritable histoire des armes}

\section{La provenance des armes et l'époque de leur introduction au Mont comme armes de saint Michel : une invention contemporaine de l'incendie de 1112}

Ainsi que l'a voulu Baudri, la Relatio est en effet le texte de référence, puisque tous les documents antérieurs susceptibles d'attester l'existence des armes et de rendre compte de leur histoire ont irrémédiablement péri dans les flammes du fameux incendie de 1112. Curieusement, seules les archives relatives aux armes de l'archange ont souffert de l'incendie. Si l'on ajoute qu'aucune autre trace de leur longue présence au Mont ne subsiste, pas même dans la Relatio qui affirme seulement que " la dévotion chrétienne les vénère humblement ${ }^{94}$ " sans fournir de preuves ni de précisions, on voit bien que la prétendue antiquité de la présence des armes au Mont ne tient pas longtemps ${ }^{95}$. Il faut donc renoncer à considérer le texte de

92. Ibid., p. 310 .

93. Inventaire de 1790, cité par J. DuBoIS, « Le trésor des reliques du Mont Saint-Michel ", op. cit., p. 512.

94. Relatio: "Que Christiana devotio suppliciter veneratur".

95. Sans compter les innombrables objections auxquelles la Relatio prête flanc (on peut se demander pourquoi Baudri, qui est archevêque de la région voisine depuis 1107, 
Baudri comme ce qu'il prétend être (l'enregistrement écrit de la déposition d'un témoin garant des traditions orales et écrites) et l'examiner seulement pour ce qu'il est réellement : le plus ancien témoignage sur l'existence d'armes miniatures de l'archange destiné à l'usage exclusif du Mont et composé à un moment où l'authenticité des petites armes et le bien-fondé de leur présence sur un autel de l'église ne fait pas l'unanimité, en particulier, semble-t-il, chez les religieux.

La conclusion du récit du prieur et les arguments et injonctions sur lesquels Baudri clôt l'ensemble de sa Relatio laissent entendre que des rumeurs discordantes devaient circuler autour de l'épée et du bouclier. Le premier, en célébrant la piété des anciens, appelle en effet implicitement à les imiter : "Nos ancêtres respectaient ces enseignes plus que nous : ils étaient plus religieux, plus mûrs, plus proches de l'époque à laquelle elles appartenaient ${ }^{96}$. "Quant à Baudri, après avoir multiplié les preuves par analogie en assimilant successivement les armes à la Manne, au manteau de l'archange et à la sainte Ampoule de Reims, il conclut sur un appel à la bienveillance des lecteurs assez singulier : "À celui qui lit sans se moquer, paix, exultation et joie qui ne soit pas éphémère. Amen ${ }^{97}$. " La seule destruction d'archives ne saurait être responsable d'une subite méfiance visà-vis de reliques à l'antiquité avérée : pourtant, après l'incendie, la nécessité se fait sentir de disposer d'un document fiable et incontestable qui explique la présence d'armes miniatures attribuées à saint Michel au Mont. L'archevêque de Dol, connu également pour son " talent de polygraphe ${ }^{98}$ ", met alors en forme les documents qu'un (des) religieux autorisé(s) - la figure anonyme et commode du prieur dénuée de réalité autre que littéraire - lui procure(nt) sur la provenance et la signification des objets en question. Puis il se porte garant de ce récit en qualité d'archevêque et de témoin. S'il n'est pas ici question de commanditaire(s) et destinataire(s) précis, alors que le texte est si manifestement destiné à l'usage exclusif du Mont, c'est que l'objet du texte, le statut et la signification armes de saint Michel, ne font pas l'unanimité dans la communauté. Dans une cer-

s'avise seulement après 1112 de l'existence des armes et de leur histoire; pourquoi, également, l'informateur de Baudri qui assure avoir "souvent vu et lu " le nom des messagers s'abstient de les donner? etc.), le rapprochement avec un autre texte de Baudri également destiné à consigner la tradition orale pour la préserver, les Actes de la translation du chef de saint Valentin (PL, t. 166, col. 1154-1161) est fort instructive. La méthode de Baudri pour retracer l'histoire de cette relique, dont la présence à Jumièges est attestée avant le texte de Baudri, est à l'opposé de celle qu'il utilise pour les armes miniatures. Il enregistre notamment tous les témoignages disponibles et ne se prive pas de fournir des éléments susceptibles de dater les miracles qu'il relate et d'en identifier les protagonistes.

96. "Maiores nostri insignia ista ampliori quam nos habuerunt reuerentie, utpote qui religiosiores, qui maturiores, qui temporibus illis fuere proximiores ".

97. "Legenti nec irridenti pax et exultatio et gaudium non transitorium. Amen."

98. Tilliette, Jean-Yves (éd., trad. et comm.), Baudri de Bourgueil, Carmina, Paris, Les Belles Lettres, 1998, t. 1, p. x.

99. Introductio monachorum : "Quomodo autem per tantum tempus isdem floruerit locus, vel quibus quantisque choruscaverit virtutibus inconitum habemus. Numquam tamen illic 
taine mesure, c'est logique, puisque les archives du Mont spécifient ${ }^{99}$ bien que la période qui suit la fondation du Mont (qui a lieu en 708 et est relatée dans la Revelatio) et précède l'arrivée des Bénédictins (en 966 et correspond à l'Introductio monachorum) n'est absolument pas documentée et qu'on ne sait rien des événements miraculeux qui s'y produisirent alors. Mais c'est surtout la taille modique des reliques qui doit poser problème. Certes, l'archange l'a justifiée en soulignant que c'étaient bien là ses armes, même si elles n'avaient bien sûr pas servi à combattre ${ }^{100}$. Baudri y insiste d'ailleurs beaucoup : elles ont vraiment l'apparence des armes utilisées par les enfants pour jouer ou s'entraîner à l'art de la guerre et c'est ce qui le mène à s'interroger sur les raisons de leur présence sur l'autel :

"Tous deux [l'épée et le bouclier] ne sauraient trouver un emploi à l'usage de la guerre mais sont seulement à l'image d'armes. Tandis que moi, Baudri, $[\ldots]$ je cherchais à découvrir et voulais scrupuleusement entendre l'origine de cette histoire [après avoir éconduit un premier interlocuteur], je fis venir le prieur du monastère et questionnais avec intérêt cet homme : que signifiait cet appareil conservé sur un autel par une si grande église ${ }^{101}$ ?"

Leur petite taille en fait des objets insolites, inhabituels et par là même sujets à caution et Baudri est investi d'une double mission : l'archevêque voisin doit se porter garant de leur authenticité, tache qui relève de sa compétence, et l'écrivain reconnu assurer et prouver cette authenticité en composant un texte imparable.

L'introduction des armes au Mont ne précède vraisemblablement que de très peu la composition du premier témoignage les concernant. L'absence de témoins antérieurs à la Relatio, ses modalités de composition, l'hostilité incrédule dont on devine que les armes sont alors l'objet ne font pas de doute là-dessus. Le prétexte invoqué à la composition de la Relatio, l'incendie de 1112, pourrait bien correspondre à la mise en place d'un culte des armes. On le sait, catastrophes et travaux sont souvent l'occasion d'inventions de reliques (aux deux sens du terme) et de redécouvertes opportunes. Le miracle De repertione sanctarum reliquiarum évoqué plus haut ${ }^{102}$ est là pour en témoigner et l'on voit bien comment l'incendie de 992 à l'origine de la redécouverte des reliques archangéliques du Gargan peut avoir été récupéré à l'occasion de celui de 1112. On aurait alors opportunément trouvé (redécouvert) dans les décombres ou au moment des travaux qui surviennent peu après des objets dépareillés, un poignard et ce qui devient l' "écusson " de saint Michel. La provenance lointaine et floue dont les affuble la Relatio est à lire comme l'aveu de l'ignorance de leur origine. L'époque mythique antérieure à l'installation des Bénédictins au Mont à laquelle on rattache leur apparition au Mont trahit une incapacité à situer

defuisse virtutum insignia a majoribus percipimus [...]. » (Dom T. LE RoY, Curieuses recherches, p. 422).

100. Cf. supra p. 165 et n. 26.

101. Relatio.

102. Cf. supra p. 168 et suiv. et n. 40 . 
dans le temps à quand remonte la présence de ces objets au Mont. Si l'on ajoute que les travaux de reconstruction du Mont auquel l'abbé Roger II (1106-1123), dont les annales du Mont célèbrent la gestion temporelle et le pragmatisme ${ }^{103}$, durent mobiliser les ressources financières de la communauté, il y a toute chance pour que les armes doivent à l'incendie non seulement le premier témoignage de leur existence mais, surtout, leur invention : promouvoir un nouveau culte a toujours été une des solutions choisies par les centres de pèlerinage pour répondre à des impératifs financiers. Le fait que les reliques miniatures soient définies aussi comme le symbole de la primauté du Mont normand sur le sanctuaire des Pouilles finit de convaincre de leur caractère récent. Il correspond en effet à une réalité historique effective non pas sitôt après la fondation du Mont normand mais à la période où Baudri écrit :

" La période du Xl ${ }^{\mathrm{e}}$ au XIII ${ }^{\mathrm{e}}$ siècle voit un renversement du rôle des deux grands sanctuaires michaeliens d'Occident, le mont Gargan et le Mont-SaintMichel. Tandis que le Gargan, célèbre durant le haut Moyen Âge, voit sa fréquentation se restreindre progressivement à l'Italie, le Mont-Saint-Michel acquiert un rayonnement international ${ }^{104}$."

\section{Le remaniement d'un document fourni par un religieux montois}

Reste à savoir à partir de quoi Baudri a élaboré son texte : il a évidemment eu accès à une documentation écrite. L'étude de l'intertexte qui nourrit le discours du prieur met d'ailleurs en évidence, outre le recours à des références scripturaires habituelles à Baudri et de nombreuses autocitations, trois types de sources écrites : le corpus montois (Revelatio, Introductio monachorum), l'Apparitio $S$. Michaelis, texte fondateur du mont Gargan ainsi que le miracle du dragon découpé en morceaux. Plus encore que la connaissance intime des textes montois, la présence diffuse de l'Apparitio ${ }^{105}$ et sa combinaison réussie avec certains éléments du miracle du dragon trahissent le religieux montois. Ceci explique, par exemple, le

103. Cf. HuYNES, op. cit., t. 1, p. 161-165.

104. ChelinI, Jean, Branthomme, Henri, «Les chemins de Dieu ", Histoire des pèlerinages chrétiens des origines à nos jours, Paris, 1982, p. 168.

105. Le récit de translation est à ce point imprégné d'éléments importés de l'Apparitio qu'il est impossible de les isoler un à un. À cet égard, le réemploi d'une phrase prononcée par l'archange lors de sa première apparition à l'évêque de Siponte est tout à fait suggestive. Dans l'Apparitio, saint Michel félicitait l'évêque et son peuple de leur initiative : "Vous avez bien fait de demander à Dieu d'éclaircir le mystère qui échappait aux hommes, la raison pour laquelle l'homme [Gargan] a été frappé par sa propre flèche. " Dans la Relatio, l'attitude du peuple déconcerté par la présence des armes miniatures s'inspire directement des propos de l'archange et de l'attitude du peuple italien : « Ils rendent grâce de ce qui s'est produit et qu'ils constatent; ils décident de s'informer de ce qu'ils ignorent encore. Ils supplient Dieu avec insistance et osent le conjurer de révéler son secret à ses suppliants. L'évêque veille toute la nuit [...] Dieu, que ce peuple touchait sa pieuse et très agréable obstination, ne put longtemps rester sourd à sa famille dévouée. " L'écho est ici amplifié et dûment combiné à une référence scripturaire (Luc 11, 5-11, la parabole de l'ami importun), ce qui en masque l'origine. 
rôle majeur joué par le personnage de l'évêque dans le texte de Baudri : alors que ce personnage n'existe pas dans le miracle du dragon, il assure dans l'Apparitio un rôle comparable ${ }^{106}$. Baudri n'est pas l'auteur de cette fusion de trois sources spécifiquement michéliennes ${ }^{107}$ mais remanie un texte qu'on lui a confié ${ }^{108}$. Contrairement aux allégations de Jean Laporte, on peut même penser qu'il croit sincèrement à l'antiquité et à l'authenticité des armes miniatures : rien dans la Relatio ne permet en tout cas de penser qu'il écrit en connaissance de cause. Peut-être faut-il même voir dans le crédit que lui accordent les Mauristes puis les érudits des XIX et $\mathrm{xx}^{\mathrm{e}}$ siècle un indice de sa bonne foi.

\section{Une mise en place contrariée et un statut ambigu (XII siècle)}

Le culte des reliques miniatures n'a pourtant pas connu un essor immédiat : contrarié par le caractère inhabituel des objets, en butte à des religieux sceptiques, il a également souffert du départ précipité de l'abbé Roger II. Cet ancien prieur de Jumièges fut en effet déposé par Henri Beauclerc en 1123 et remplacé par un piètre gestionnaire, Richard de Mère, à son tour rapidement prié de regagner son monastère d'origine (1127). Quand arrive le milieu du XII ${ }^{\mathrm{e}}$ siècle et l'abbatiat de Robert de Torigni (11541186), le culte des reliques en est toujours au même point et ne s'est enrichi d'aucune tradition ou témoignage susceptibles de montrer leur efficacité. Guillaume de Saint-Pair en est réduit, dans son Roman, à reproduire la Relatio sans y ajouter quoi que ce soit qui compense ses imprécisions ou enregistre le développement du culte de ces reliques. Surtout, il n'emploie jamais pour désigner les armes archangéliques le terme de "reliques " mais traduit sytématiquement le polysémique insignia qu'on trouve à plusieurs reprises chez Baudri par " enseignes ". Certes, il préserve ainsi les jeux de mots de Baudri sur la "signification " de ces " enseignes ", mais il occulte le sens de " choses insignes ", " signes ", " reliques " que renferme aussi cet insignia. Quelque trente-cinq ans après Baudri, les armes de saint Michel n'ont donc pas encore véritablement trouvé le rang de reliques incontestables que la Relatio visait à leur faire reconnaître. Le choix de s'en remettre prudemment à l'autorité compétente et inattaquable de Baudri suggère au contraire le désir de relancer un culte tombé en déshérence avant même d'avoir connu un essor notable.

\footnotetext{
106. Dans les deux cas, Apparitio (AS, Sept., VIII, p. 61-62) et Relatio, le peuple désemparé se tourne vers lui, dans les deux cas, il est à l'instigation des trois jours de jeûne également absents du miracle du dragon - couronnés par l'apparition de saint Michel qui s'adresse à lui pour donner la clef d'événements miraculeux et incompréhensibles aux hommes et indiquer sa volonté, etc.

107. D'autant que le miracle du dragon semble n'avoir connu qu'une diffusion restreinte (seuls trois témoins subsistent).

108. Il vient d'ailleurs de remanier l'Historia Hierosolymitana et refond dans les années 1120 la Vita S. Hugonis pour l'abbaye de Jumièges dont Roger II a été prieur avant d'être à la tête du Mont-Saint-Michel.
} 


\section{Des reliques qu'on popularise (XIV'e siècle)}

Effet de sources ou reflet d'une réalité historique, on ne sait ce qu'il advint de cette tentative avant le milieu du XIv ${ }^{\mathrm{e}}$ siècle, époque à laquelle les petites armes ont gagné, pour plusieurs siècles, le statut de reliques. L'Inventaire de $1396{ }^{109}$ qui recense les différentes pièces du Trésor du Mont et les rubrique sous le $n^{\circ} 28$ est explicite. Cette fois le terme de "relique " (pignus) est utilisé, conjointement à celui de "signe, preuve " (intersignum) pour évoquer les armes : "Item, nous avons ce qui est à la fois un signe et une relique précieux, l'écu et le glaive ou épée utilisés pour tuer le dragon sur un ordre divin par S. Michel et miraculeusement transféré dans ce saint endroit $[\ldots]^{110}$. " Cette reconnaissance officielle accompagne un succès plus populaire et le Mystère ${ }^{111}$, en proposant une version qui s'écarte de l'originale et est mise en scène à l'intention des pèlerins, rend compte de la vitalité du culte des reliques. La nouvelle provenance géographique qu'on leur attribue maintenant et l'existence de lamelles de cuivre expliquant notamment l'origine irlandaise des armes n'y sont sans doute pas étrangers.

\section{Diffusion du culte des reliques ( $X V^{e}$ siècle)}

Si les armes miniatures se sont imposées comme reliques au Mont, elles n'ont pas laissé de souvenir ailleurs avant le milieu du Xve siècle. C'est, paradoxalement, le cantique allemand $(1457)^{112}$, dans lequel il n'est pas fait mention des armes, qui exporte une partie de l'histoire jadis consignée par Baudri. L'attention qu'accorde la communauté montoise aux armes miniatures est alors à son comble : au début du siècle, on recopie le texte intégral de la Relatio dans les ms 211 et 213 et on copie le Mystère; plus tard, on rédige une version abrégée de la Relatio, le De scuto et ense, accompagnée dans le même manuscrit (ms 212) de son pendant en français ${ }^{113}$. En même temps qu'on met à disposition du public des versions tronquées ou divergentes de l'originale, on s'emploie à conserver le plus ancien témoignage qu'on réserve à l'usage interne de la communauté. Les armes miniatures semblent alors bénéficier des retombées de la grande affluence de pèlerins au Mont. C'est en effet l'époque qui coïncide avec l'essor de la figure de saint Michel " comme saint royal et national ${ }^{114}$ ". Amorcé sous

109. C'est également probablement le premier inventaire effectué au Mont. DuBors, Jean, "Le trésor des reliques de l'abbaye du Mont Saint-Michel ", Millénaire Monastique du MontSaint-Michel, t. 1, Paris, Lethielleux, 1967.

110. BM Avranches, $\mathrm{ms} 213, \mathrm{f}^{\circ} 158 \mathrm{v}^{\circ}-162$, sous le $\mathrm{n}^{\circ} 28$ : «Item, habemus preciosum et intersignum et pignus, scutum cum gladio uel ense supra drachonem precepto diuino a beato Michaele interfectum, et ad hunc sanctum locum miraculose delatum, ut patet ex narratione domini Baldrici, Dolensis archiepiscopi, intus reseruata. "Cité par Jean DuBors, op. cit., p. 569. 111. Supra p. 166-169.

112. Supra p. 169-170.

113. Supra p. 171-172.

114. BEAune, Colette, Naissance de la nation France, Paris, Gallimard, 1985, réed. 1993, « coll. Folio histoire; n ${ }^{\circ} 56$ ", p. 264. 
Charles VI (1380-1422) qui vouait à saint Michel un culte particulier - mais non privilégié - et effectif sous Charles VII (1422-1461), le passage de la France sous la protection de saint Michel est officialisé par Louis XI (14611483) qui créa notamment l'ordre de saint Michel (1469). L'archange en armes terrassant le dragon s'affiche alors sur les étendards ${ }^{115}$ et symbolise la résistance à l'ennemi anglais identifié au dragon vaincu puis la reconquête : on conçoit que les reliques, même miniatures, d'un combat où l'archange extermine un dragon insulaire aient pu alors faire recette...

\section{Stagnation? (XVI ${ }^{e}$ siècle)}

Le $\mathrm{XVI}^{\mathrm{e}}$ siècle n'a laissé qu'un seul témoignage relatif aux armes, celui de l'évêque d'Avranches Robert Cenalis (1532-1566) ${ }^{116}$ qui suggère que les reliques rencontrent à nouveau certaines difficultés, si l'on en croit le préambule explicatif adressé au lecteur qu'il juge indispensable de fournir avant de raconter l'histoire des reliques ${ }^{117}$. Il est vrai que l'abbaye du MontSaint-Michel a alors largement entamé sa décadence et ne vit plus que sur son prestige passé. La vie régulière est presque complètement abandonnée, et, avant l'arrivée des Mauristes, "l'ignorance y étoit si grande que plusieurs ne pouvoient pas même lire du françois " et il arrive que les pèlerins désireux de voir les reliques soient obligés d'aller chercher "le thresorier en un cabaret " pour s'entendre dire de " cet ivrogne " : " Je me donne au diable si les chiens ne les ont mangez [les reliques]. " L'anecdote contée par Dom De Camps ${ }^{118}$ résume assez bien la situation : malgré la décadence de la vie régulière, le sanctuaire attire toujours les pèlerins, mais sans doute l'affluence est-elle moins massive qu'au siècle précédent.

\section{Dernier soubresaut avant la disparition (XVII et XVIII siècles)}

On ne retrouve à nouveau une grande concentration de témoins qu'au XVII ${ }^{\mathrm{e}}$ siècle ${ }^{119}$, notamment avec le renouveau spirituel et intellectuel qu'assure au Mont l'installation de la Congrégation de Saint-Maur (1622). Cette fois encore, comme au $\mathrm{XV}^{\mathrm{e}}$ siècle mais dans une moindre mesure, la diffusion de la tradition locale à l'extérieur du Mont dévoie l'original, tandis qu'au Mont même elle est l'objet d'un mouvement de retour aux sources. Les reliques miniatures figurent alors en bonne place dans le trésor du

115. Sous le règne de Charles VII, ibid., p. 264-265.

116. CEnAlis, Robert, Neustriae Hierarchia, 1660. Cf. Annexe.

117. Op. cit., $\mathrm{f}^{\circ} 21$ sq., où il explique qu'il est absurde de vouloir voir dans les armes (dont il ne précise pas qu'elles sont miniatures) exposées au Mont des armes utilisées pour vaincre un mauvais esprit (" ad explodendum cacodemonum "), puisqu'il s'agit en fait de signes concrets destinés à témoigner pour la postérité d'actions invisibles ( " celestes spiritus beatissimi inuisibiles suas actiones sensibili aliquo indicio, ad perpetuam rei memoriam posteris prodant").

118. Histoire générale, t. 1, p. XLVI.

119. Cf. Annexe. 
Mont. Elles en sont même, si l'on en juge par l'importance que leur accorde le livret de pèlerinage de Feuardent, l'un des fleurons.

Pourtant, en $1744^{120}$, signe des temps, elles ne conservent plus rien de ce statut de reliques qui leur fut tardivement reconnu. Rayées de l'inventaire d'un trésor maintenant réduit à sept pièces majeures, elles retrouvent peut-être leur destination première puisque les voilà considérées comme des ex-voto venus d'Irlande. Si lui ne sait pas qu'elles sont enfermées dans les armoires où l'on entasse les reliques, la Révolution se chargera de les dénicher pour les faire disparaître définitivement.

Ainsi, après avoir non sans mal acquis le statut de reliques de l'archange, les petites armes guerrières, sans doute au départ d'obscurs exvoto dissociés l'un de l'autre, ont-elles finalement retrouvé leur destination première. Les difficultés auxquelles se sont heurtés les promoteurs de ce culte lors de son lancement au début du XII ${ }^{\mathrm{e}}$ siècle ne sont pas seulement conjoncturelles (départ de l'abbé, remplacé par un autre abbé contraint de partir à son tour, hostilité interne...) : elles tiennent surtout à la nature même des objets. Peu importe qu'il s'agisse d'armes, au contraire même, puisque le XII ${ }^{\mathrm{e}}$ siècle est riche en reliques guerrières ${ }^{121}$. Trois facteurs paraissent expliquer leur lancement raté. Leur petite dimension qui les apparente à des jouets a manifestement nui d'abord au premier essor de leur culte. Le fait qu'il s'agisse d'une paire indissociable alors qu'il n'y pas d'unité de facture et que rares sont les reliques doubles n'a pas dû arranger les choses. Enfin, la complexité de leur signification ne pouvait, dans un premier temps, qu'entraver la diffusion de leur culte : ce ne sont pas les armes utilisées par saint Michel dans un de ses combats, mais une preuve concrète laissée par saint Michel de ce qu'il a combattu invisiblement. L'épée et le bouclier miniatures réunissaient donc trop de caractéristiques inhabituelles pour susciter un engouement spontané. Aussi s'est-on assuré la collaboration de Baudri, archevêque voisin et écrivain de renom. En dépit de son contenu et de sa forme irréprochables, dans un premier temps, la Relatio échoue à remplir sa fonction et ce n'est qu'au XIV ${ }^{\mathrm{e}}$ siècle qu'on devine que les reliques guerrières sont en faveur au Mont.

L'examen du dossier des armes miniatures permet donc de restituer partiellement quelques étapes de leur carrière au Mont et surtout met fin à l'idée répandue qu'elles ont connu un culte continu à partir de leur introduction. Quoique l'exercice ait ses limites, puisque de nombreuses questions demeurent en suspens, il est aussi l'occasion de mieux saisir les modalités de transmission d'une tradition à partir d'un texte unique (la Relatio).

120. Ibid.

121. Nous devons cette remarque, ainsi que les exemples suivants, à Florian Mazel que nous remercions. On peut penser à la sainte Lance de Longin (dont Baudri relate d'ailleurs l'invention dans son Historia Hierosolymitana) ou à l'épée de Guillaume de Gellone, dans le midi de la France. 


\section{Annexe - Chronologie et bibliographie des témoins jusqu'à la disparition des armes (1790)}

Vers 1120 : BAUDRI DE BOURGUEII, Relatio de scuto et gladio sancti Michaelis (BHL 5953) BM Avranches, $\mathrm{ms} 211, \mathrm{f}^{\circ} 26-31 \mathrm{v}^{\circ}$ et $213, \mathrm{f}^{\circ} 150-153$.

Recension brève sous le titre De scuto et ense dans le ms $212, \mathrm{f}^{\circ} 6 \mathrm{v}^{\circ}-10$. La seule édition actuellement disponible, celle de Eugène ROBILLARD DE BEAUREPAIRE d'après Dom Huynes, est lacunaire et parfois fautive : Histoire générale de l'abbaye du Mont-Saint-Michel au péril de la mer, publiée pur la première fois avec une introduction et des notes, par E. ROBILLARD DE BEAUREPAIRE, Eugène, Rouen, 1872-73, t. I, p. 137-146.

Traduction française sur le texte de Robillard de Beaurepaire : DuPont, E., Le Mont Saint-Michel et les pays étrangers. Angleterre, Allemagne, Belgique, Bruxelles, 1902, p. 2-13, reprise dans DuPONT, E., "Les pèlerinages d'enfants allemands au Mont Saint-Michel ( $\mathrm{Xv}^{\mathrm{e}}$ siècle). Le récit de Baudry, archevêque de Dol ", Annales de la société historique et archéologique de l'arrondissement de Saint-Malo, 1906, p. 38-45.

Vers 1155 : Guillaume de SaINT-PaIR, Le Roman du Mont Saint-Michel, v. 3170-3531 Michel, Francisque (éd.), " Le Roman du Mont Saint-Michel par Guillaume de Saint-Pair, poète anglo-normand du XII siècle ", Mémoires de la société des Antiquaires de Normandie, t. XX, 1853, p. 509-573

BougY, C., "Le billet savant ", Bulletin de l'OUEN, n 11, janvier 2001, p. 11-12.

\section{vers 1350-1400 : MYSTERE anonyme}

Robillard de BeAurePAIRE, Eugène, Les miracles du Mont Saint-Michel. Fragment d'un mystère du XIV siècle, Avranches, Auguste Anfray, 1862. Scène relative à l'écu et l'épée, p. 9-15.

1396 : INVENTAIRE DES RELIQUES de $1396, n^{\circ} 28$

BM Avranches, ms $213, \mathrm{f}^{\circ} 158 \mathrm{v}^{\circ}-162$, sous le $\mathrm{n}^{\circ} 28$ : «Item, habemus preciosum et intersignum et pignus, scutum cum gladio uel ense supra drachonem precepto diuino a beato Michaele interfectum, et ad hunc sanctum locum miraculose delatum, ut patet ex narratione domini Baldrici, Dolensis archiepiscopi, intus reseruata ». (Cité par DuBoIs, J. (Dom), "Le trésor des reliques de l'abbaye du Mont Saint-Michel ", Millénaire Monastique du Mont Saint-Michel, t. 1, Paris, Lethielleux, 1967, p. 569.)

\section{7 : Chant DE PELERINAGE allemand}

DuPONT, Étienne, "Les pèlerinages d'enfants allemands au Mont Saint-Michel ( $\mathrm{xv}^{\mathrm{e}}$ siècle). Le récit de Baudry, archevêque de Dol ", Annales de la société historique et archéologique de l'arrondissement de Saint-Malo, 1906, p. 19-60, chant p. 34-35.

1457-1480 : ANonyme, B. M Avranches, ms 212, De scuto et ense (BHL 5953b) et De l'escu et l'espée saint Michiel

$\mathrm{f}^{\circ} 6 \mathrm{v}^{\circ}-10$, De scuto et ense, recension brève du texte de Baudri, copiée - et probablement composée - sous l'abbatiat de Guillaume d'Estouteville (1446-1483), après 1457.

$\mathrm{f}^{\circ}$ 49-54:De l'escu et l'espée saint Michiel : translation en français du De scuto et ense. 


\section{Vers 1560 : Cenalis (CENEAU) Robert}

Hierarchia Neustriae Authore Reuerendo in Christo patre Roberto Cenali..., BNF lat. $5201, \mathrm{f}^{\circ} 21 \mathrm{sq}$.

\section{4 : FeuARDENT, François}

Histoire de la Fondation de l'Eglise et Abbaie du Mont S. Michel pres celui de tombe : \& des miracles, reliques, \& indulgences donnez en icelle. Tout recueilli des Archives du dit lieu, Constances, Jean Le Cartel, 1604, p. 27-41.

\section{2 : Naveau, Michel}

Chronicon apparitionum \& gestorum S. Michaelis Archangeli a mundo condito ac deinceps per varias orbis partes in sex libros distinctum cum summarium ad finem operis librorum \& capitum \& tabula rerum memorabilium, Douai, 1632, lib. III, cap. XXIII, p. 213.

\section{3 : Du MONSTIER, Arthur}

Neustria Pia seu de omnibus et singulis abbatiis et prioratibus totius Normaniae, Rouen, J. Berthelin, 1663, ch. III, p. 379-381.

\section{0 : HuYNES Jean (Dom)}

Histoire générale de l'abbaye du Mont-Saint-Michel au péril de la mer publiée pur la première fois avec une introduction et des notes, par Eugène ROBILLARD DE BEAUREPAIRE, Rouen, 1872-73, 2 vol., t. I, p. 42-43 et 135-148.

BNF ms fr. 18947 et 18948.

\section{8 : LE Roy Thomas (Dom)}

Les curieuses recherches du Mont Saint-Michel, ROBILLARD DE BEAUREPAIRE éd., Caen, 1878, 2 vol, t. 1, p. 87 et 141.

\section{7 : Boudon HENRY-MARIE}

La dévotion aux neufs chœurs des saints anges, et en particulier aux saints anges gardiens, Paris, 1755, p. 294-300.

\section{4 : ANONYME}

Histoire du Mont-Saint-Michel Depuis sa fondation Par S. Aubert en 708 Jusqu'à l'Année 1744. composée Par un Religieux Benedictin de L'Abbaye Royalle du MontSaint-Michel De la Congrégation de S. Maur, BNF ms fr. 18949, p. 25.

\section{0 : INVENTAIRE de 1790}

Dubors, Jean (Dom), «Le trésor des reliques de l'abbaye du Mont-Saint-Michel ", Millénaire Monastique du Mont Saint-Michel, t. 1, Paris, Lethielleux, 1967, p. 512. 


\section{RESUME}

Le grand sanctuaire michélien occidental, l'abbaye bénédictine du Mont Saint-Michel, a longtemps conservé dans son Trésor un glaive et un bouclier miniatures qui passaient pour avoir été légués par l'archange lui-même. Prétendûment introduites à l'époque mythique des chanoines (708-966), ces petites armes ont définitivement disparu à la Révolution (1790). L'examen des différents états de la tradition écrite relative à ces reliques singulières permet de préciser leur date d'introduction et l'évolution de leur statut au Mont, tout en mettant à jour le rôle déterminant du premier texte qui leur est consacré, la Relatio de scuto et gladio $S$. Michaeli, composée au début du XII ${ }^{\mathrm{e}}$ siècle par Baudri de Bourgueil.

\section{ABSTRACT}

The great occidental Michaelian sanctuary, the Benedictine abbey of Mont Saint Michel, has kept for a long time in its treasury a sword and a miniature shield which were supposed to have been bequeathed by the archangel himself. It is believed that these small weapons were put in the treasury during the mythic period of the canons (708-966) before disappearing in the French Revolution (1790). The study of the different stages of the written tradition relating to these remarkable relics allows us to specify the date of their introduction and evolution of their status to the Mont Saint Michel. It enables us to also re-determine the major role of the first text dedicated to them, Relatio de scuto et gladio S. Michaeli, written at the beginning of the $12^{\text {th }}$ century by Baudri de Bourgueil. 
